\title{
Asymptotic Flatness and Bondi Energy in Higher Dimensional Gravity
}

\author{
Stefan Hollands* \\ Enrico Fermi Institute, Department of Physics, \\ University of Chicago, 5640 Ellis Ave., \\ Chicago IL 60637, USA \\ Akihiro Ishibashi ${ }^{\dagger}$ \\ Department of Applied Mathematics and Theoretical Physics, \\ Centre for Mathematical Sciences, \\ University of Cambridge, Wilberforce Road, \\ Cambridge CB3 0WA, UK
}

February 7, 2008

\begin{abstract}
We give a general geometric definition of asymptotic flatness at null infinity in $d$-dimensional general relativity ( $d$ even) within the framework of conformal infinity. Our definition is arrived at via an analysis of linear perturbations near null infinity and shown to be stable under such perturbations. The detailed fall off properties of the perturbations, as well as the gauge conditions that need to be imposed to make the perturbations regular at infinity, are qualitatively different in higher dimensions; in particular, the decay rate of a radiating solution at null infinity differs from that of a static solution in higher dimensions. The definition of asymptotic flatness in higher dimensions consequently also differs qualitatively from that in $d=4$.

We then derive an expression for the generator conjugate to an asymptotic time translation symmetry for asymptotically flat spacetimes in $d$-dimensional general relativity ( $d$ even) within the Hamiltonian framework, making use especially of a formalism developed by Wald and Zoupas. This generator is given by an integral
\end{abstract}

*Electronic mail: stefan@bert.uchicago.edu

${ }^{\dagger}$ Electronic mail: A.Ishibashi@damtp.cam.ac.uk 
over a cross section at null infinity of a certain local expression and is taken to be the definition of the Bondi energy in $d$ dimensions. Our definition yields a manifestly positive flux of radiated energy.

Our definitions and constructions fail in odd spacetime dimensions, essentially because the regularity properties of the metric at null infinity seem to be insufficient in that case. We also find that there is no direct analog of the well-known infinite set of angle dependent translational symmetries in more than 4 dimensions.

\section{Introduction}

Theories attempting to unify the forces often require a higher dimensional spacetime, and accordingly have different properties than 4-dimensional theories. Still important and fundamental even in higher dimensional theories are the notion of an isolated system and associated conserved quantities, such as the total energy of the system.

In 4-dimensional general relativity, there exist two satisfactory notions of the total energy of a spacetime representing an isolated system, namely the "Arnowitt-Deser-Misner" (ADM) energy [1] and the "Bondi" energy [2, 3, 4]. The ADM energy represents the energy of the system "once and for all" and is mathematically given by an integral of a quantity associated with the gravitational field, over a sphere at spatial infinity. The Bondi energy measures the total energy of the system "at an instant of time", and is mathematically given by an integral over a spacelike cross section at null infinity. Thus, while the ADM energy is just a number, the Bondi energy is in general a function of time in the sense that it depends on the chosen cross section at null infinity. The difference between the Bondi energies at two different times represents the flux of gravitational radiation through the portion of null infinity bounded by the corresponding two cross sections.

While the expression for the ADM energy of a spacetime is readily generalized to an arbitrary number of spacetime dimensions, this is not so for the Bondi energy. To our knowledge, no expression for the Bondi energy or other quantities associated with the group of asymptotic symmetries has been given in the literature for more than 4 dimensions $^{1}$, let alone a systematic derivation. This is maybe not so surprising since constructions associated with null infinity tend to be more complicated in nature than corresponding constructions at spatial infinity, which may e.g. be appreciated from the fact that a completely satisfactory definition of quantities associated with asymptotic symmetries in 4 dimensions was not given as late as the early 80's [6]. The purpose of the present article is to derive an expression for the Bondi energy and momentum (Bondi energy, for short) in spacetimes of arbitrary (even) dimension.

The basic issue that needs to be settled in order to even get started on a definition of

\footnotetext{
${ }^{1} \mathrm{~A}$ generalization of the Bondi-Metzner-Sachs group to higher dimensions has been discussed in the context of supergravity [5]. The definition of asymptotic flatness given in that paper differs from the definition given in the present paper.
} 
Bondi energy in higher dimensions is to specify what exactly one means by the statement that a spacetime represents an "isolated system". Roughly speaking, an isolated system is a spacetime that looks like Minkowski space "far away". Of course, one has to explain exactly what one means by "far away", and one must determine the precise asymptotics that should reasonably be imposed on the gravitational field at null infinity. What are the asymptics for the gravitational field in $d$ dimensions? For the sake of definiteness, suppose one would attempt to define an isolated system to be a spacetime whose metric has the form of the Schwarzschild metric (in suitable coordinates), plus higher order terms in $1 / r$ as one goes off to infinity along a null direction. In other words, suppose one were to define asymptotic flatness at null infinity in the same way which works at spatial infinity. Then one would effectively eliminate from consideration all spacetimes that contain gravitational radiation through null infinity, which are of course precisely the spacetimes that one wants to describe in the first place. On the other hand, if one imposes drop off conditions that are to weak, then it will in general be impossible define a notion of radiated energy of such a spacetime in a meaningful way. Thus, the task is to find a definition of asymptotic flatness that is both general enough so as to allow sufficiently many physically interesting radiating spacetimes, and stringent enough so as to allow one to derive meaningful expressions for the energy, as well as possibly other quantities associated with asymptotic symmetries.

The original definition of asymptotic flatness in 4 dimensions proposed by Bondi and collaborators [2, 4] was formulated in terms of detailed conditions on the metric components in a preferred coordinate frame and was arrived at via a study of gravitational waves near infinity. Their definition was later elegantly recast into the language of "conformal infinity" following the work of Penrose [7, 8, 9, 10, 11. In this language, a spacetime is said to be asymptotically flat, if it can be conformally embedded into a smooth "unphysical" spacetime via a conformal transformation with conformal factor $\Omega$, so that the points at infinity are at the "finite" location $\Omega=0$ in the unphysical spacetime, and so that the gradient of the conformal factor $\Omega$ is null there. The main arguments that have been advanced in favor of this definition are that it covers the known exact solutions of Einstein's equation that one intuitively thinks of as representing isolated systems, and that the definition can be proven to be stable under linear perturbations [12, in the sense that any compactly generated solution to the linearized equations of motion around an asymptotically flat solution satisfies the linearized version of the above definition of asymptotic flatness in a suitable gauge ${ }^{3}$.

Thus, the first task of our paper is to obtain an appropriate generalization of asymp-

\footnotetext{
${ }^{2}$ Other, less restrictive notions of an isolated system may also be considered, for example systems that look like a Kaluza-Klein space far out in the "non-compact directions". However, the analysis of such metrics and of the associated conserved quantities would be substantially different from the ones studied in the present paper.

${ }^{3}$ By contrast, if one were to adopt the e.g. same definition of asymptotic flatness at null infinity as one has at spatial infinity, then such a definition would not be stable under linear perturbations.
} 
totic flatness to higher dimensions. This definition will be motivated as in 4 dimensions by a detailed analysis of the decay properties of linear perturbations at null infinity. We show that perturbations typically drop off as $1 / r^{(d-2) / 2}$ as one approaches null infinity, which, as we note, differs from the drop off rate of the Schwarzschild metric, $1 / r^{d-3}$, when the spacetime dimensions is greater than 4. The appearance of half odd integer powers of $1 / r$ in the tail of the metric at null infinity in odd $d$ implies in particular that the unphysical metric will not be smooth at null infinity. It turns out that, for this reason, a geometrical definition of null infinity as given above for $d=4$ does not appear to be possible in odd spacetime dimensions. We will therefore restrict our attention to spacetimes of even dimension in this paper. We will also see that the detailed behavior of the perturbations near null infinity differs qualitatively from that in 4 dimensions in that the trace of the metric perturbation drops off one power in $1 / r$ faster than the perturbation itself $^{4}$. Consequently, our definition of asymptotic flatness in $d>4$ dimensions also differs qualitatively from that in 4 dimensions in that it involves, for example, additional conditions on the metric volume element as one approaches null infinity.

The mathematical expression for the ADM energy, including the correct normalization, can be derived (in arbitrary dimension) in a simple and straightforward manner within the Hamiltonian framework of general relativity where it is seen to represent the "charge" conjugate to an infinitesimal asymptotic time translation at spatial infinity [13. It was shown by Wald and Zoupas 14] (based on earlier work by Ashtekar and Streubel [11, see also [15]) that an expression for the Bondi energy in 4 dimensional general relativity can also be arrived at within a Hamiltonian framework as the quantity conjugate to an asymptotic time translation at null infinity, although the situation is certainly considerably more complicated compared to spatial infinity. This expression was shown to be unique under some natural assumptions and agrees with the previously known one [10, 6]. The formalism of [14] is in fact capable of dealing with arbitrary diffeomorphism covariant theories of gravity (in arbitrary dimension) in the presence of boundary conditions at null-surfaces. We employ it here to establish the existence and uniqueness of a generator conjugate to an asymptotic time-translation in $d$-dimensional vacuum general relativity ( $d$ an even number) within the context of our asymptotic flatness condition, and we take this generator as the definition of the Bondi energy in $d$ dimensions. The algorithm by Wald and Zoupas specifies this generator only indirectly via its variation under a suitable variation of the spacetime metric, so further work is required to actually find a local expression for this quantity. Such an expression is provided in eq. (100). As in 4 dimensions, our definition of the Bondi energy has the property that it yields a manifestly positive flux of energy given by the square of a suitably defined "news tensor".

We emphasize that the issue of existence of a generator conjugate to an asymptotic

\footnotetext{
${ }^{4}$ As we will show, this phenomenon is closely related to the fact that the transverse traceless gauge is regular at null infinity in $d>4$, whereas this is not the case in $d=4$, where the so-called "GerochXanthopoulos" gauge has this property.
} 
time translation symmetry (or a more general other asymptotic symmetry) is by no means automatic but rather depends crucially on the nature of the boundary conditions. As a rule, boundary conditions that are "too weak" will prohibit the existence of a generator. In our case, the boundary conditions are independently determined by a perturbation analysis, and therefore not put in "by hand" in order to guarantee the existence of a generator. That we find existence of a generator is therefore a consistency check.

The case $d=4$ seems to be "exceptional" with regard to many of our constructions from the point of view of general $d$. For example, the definition of the news tensor differs in dimensions greater than 4. Also, while the unphysical Weyl tensor can be proven to vanish one order faster than the unphysical Ricci tensor in $d=4$, this does not appear to be the case in higher dimensions. On the other hand, the unphysical Ricci scalar vanishes one order faster than the unphysical Ricci tensor itself in $d>4$, while both quantities generically have the same drop off behavior in $d=4$. In $d=4$, it is well known that the asymptotic symmetries form an infinite dimensional group which comprises, besides the transformations corresponding to the usual symmtries of Minkowski spacetime, an additional infinite set of (mutually commuting) "angle dependent translations", sometimes called "supertranslations" 5 . We find that there is no direct analog of the supertranslations in $d>4$. Another curious feature, which is of a more technical nature but nevertheless seems to underly many of the differences between $d=4$ and higher dimenions is that, while linear perturbations can be proven to be regular at null infinity in the transverse traceless gauge in $d>4$, this is not so in $d=4$, where one has to choose the (somewhat complicated) Geroch-Xanthopoulos gauge [12.

The contents and main results of this paper may now be summarized. In section 2 we present our definition of asymptotic flatness in arbitrary dimension and define the notion of an asymptotic symmetry. In section 3 we show that our definition of asymptotic flatness is stable under linear perturbations, provided that the perturbations are in a suitable gauge. In section 4 we recall the general formalism of 14 for defining generators associated with asymptotic symmetries, and in section 5 we derive our formula for the Bondi energy. We also verify that our expression for the Bondi energy agrees with the expected one (i.e., the ADM energy) in the $d$ dimensional analogue of the Schwarzschild metric. We draw our conclusions in section 6. For simplicity, we restrict attention to the case of vacuum general relativity throughout this article. Some remarks concerning the incorporation of matter fields are also given in section 6.

Our conventions are the same as in [16]: The signature of the metric is $(-++\ldots)$, the convention for the Riemann tensor is $\nabla_{[a} \nabla_{b]} k_{c}=(1 / 2) R_{a b c}{ }^{d} k_{d}$ and $R_{a b}=R_{a c b}{ }^{c}$ for the Ricci tensor. Indices in parenthesis are symmetrized and indices in brackets are antisymmetrized.

\footnotetext{
${ }^{5}$ There is no relation with supersymmetry.
} 


\section{Asymptotic flatness in $d$ dimensions}

Asymptotic conditions in field theory require the specification of a background configuration and the precise rate at which this background is approached. In the case of asymptotic flatness in higher dimensional general relativity, the background is the Minkowski metric ${ }^{6}$. In order to specify the precise rate at which Minkowski spacetime is approached at null infinity, it is of great technical advantage to work within a framework in which "infinity" is attached as additional points to the spacetime manifold, $\tilde{M}$ (thereby obtaining an "unphysical" spacetime manifold $M$ ), and in which these points are brought metrically to a finite distance by rescaling the physical metric, $\tilde{g}_{a b}$, by a conformal factor $\Omega^{2}$ with suitable properties. The asymptotic flatness conditions are then formulated in terms of this rescaled "unphysical metric,"

$$
g_{a b}=\Omega^{2} \tilde{g}_{a b},
$$

and its relation to the likewise conformally rescaled version of Minkowski spacetime,

$$
\bar{g}_{a b}=\Omega^{2} \tilde{\eta}_{a b}
$$

We will refer to $\left(\bar{M}, \bar{g}_{a b}\right)$ as the "background geometry". As it is well-known, eq. (2) can be realized e.g. by conformally embedding Minkowski spacetime into a patch of the Einstein static universe $\mathbb{R} \times S^{d-1}$ with line element $\mathrm{d} \bar{s}^{2}=-\mathrm{d} T^{2}+\mathrm{d} \psi^{2}+\sin ^{2} \psi \mathrm{d} \sigma^{2}$. Here, $\mathrm{d} \sigma^{2}$ is the line element of the unit round $(d-2)$-dimensional sphere, and $\psi$ is the azimuthal angle of $S^{d-1}$. In these coordinates, Minkowski spacetime corresponds to the region $\bar{M}=\{-\pi<T \pm \psi<\pi, \psi>0\}$ of $\mathbb{R} \times S^{d-1}$, see appendix B for further details of this conformal embedding, as well as the specific form of $\Omega$.

The conformal infinity of Minkowski spacetime is the boundary of the region $\bar{M}$ in the Einstein static universe. It is divided into the five parts (1) future timelike infinity (the point $T=\pi, \psi=0$ ) (2) past timelike infinity (the point $T=-\pi, \psi=0$ ) (3) spacelike infinity (the point $\psi=\pi, T=0$ ) (4) past null-infinity (the points $T=-\pi+\psi$ for $0<\psi<\pi$ ) and (5) future null infinity (the points $T=\pi-\psi$ for $0<\psi<\pi$ ). The conformal factor $\Omega$ is smooth in a neighborhood of null infinity and vanishes there, and the gradient if $\Omega$ is null there.

Our definition of asymptotic flatness consists in specifying the precise rate at which $g_{a b}$ approaches $\bar{g}_{a b}$ as one approaches the boundary $\partial \bar{M}$. To quantify how various tensor behave at that boundary, we introduce the following notion: We will say that a tensor field, $L_{a b \ldots c}$, is "of order $\Omega^{s}$ " with $s \in \mathbb{R}$, written $L_{a b \ldots c}=O\left(\Omega^{s}\right)$, if the tensor field $\Omega^{-s} L_{a b \ldots c}$ is smooth at the boundary. It is a consequence of this definition that if $L_{a b \ldots c}$ is of order $s$ then $\Omega^{r} L_{a b \ldots c}$ is of order $s+r$, and that $\bar{\nabla}_{d_{1}} \cdots \bar{\nabla}_{d_{k}} L_{a b \ldots c}$ is of order $s-k$.

We now state our definition of asymptotic flatness in even spacetime dimensions $d>4$. (From now on, $d$ is taken to be an even number unless stated otherwise.) Let $\left(\bar{M}, \bar{g}_{a b}\right)$ be

\footnotetext{
${ }^{6}$ Other asymptotic conditions would of course require a different background.
} 
the background geometry described above. A $d$-dimensional spacetime $\left(\tilde{M}, \tilde{g}_{a b}\right)$ will be said to be weakly asymptotically simple at null infinity if the following is true:

1. It is possible to attach a boundary, $\mathscr{I}$, to $\tilde{M}$ such that any open neighborhood of $\mathscr{I}$ in $M=\tilde{M} \cup \mathscr{I}$ is diffeomorphic to an open subset of the manifold $\bar{M}$ of our background geometry, and such that points of $\mathscr{I}$ get mapped to (possibly a subset of) the boundary of $\bar{M}$ under this identification.

2. One has, relative to our background metric $\bar{g}_{a b}$, that

$$
\bar{g}_{a b}-g_{a b}=O\left(\Omega^{\frac{d-2}{2}}\right), \quad \bar{\epsilon}_{a b \ldots c}-\epsilon_{a b \ldots c}=O\left(\Omega^{\frac{d}{2}}\right),
$$

where $\bar{\epsilon}_{a b \ldots c}$ and $\epsilon_{a b \ldots c}$ denote the volume element (viewed as $d$-forms) associated with the metrics $\bar{g}_{a b}$ respectively $g_{a b}$, as well as

$$
\left(\bar{g}^{a b}-g^{a b}\right)(\mathrm{d} \Omega)_{a}=O\left(\Omega^{\frac{d}{2}}\right), \quad\left(\bar{g}^{a b}-g^{a b}\right)(\mathrm{d} \Omega)_{a}(\mathrm{~d} \Omega)_{b}=O\left(\Omega^{\frac{d+2}{2}}\right),
$$

where $g^{a b}$ is the inverse of $g_{a b}$ and where $\bar{g}^{a b}$ is the inverse of $\bar{g}_{a b}$.

It is important to note that, while our definition of an asymptotically flat spacetime is formulated relative to a specific background geometry, our definition is actually independent of the precise way in which the Minkowski metric $\tilde{\eta}_{a b}$ is written as $\Omega^{-2} \bar{g}_{a b}$ in terms of a background metric (smooth at $\mathscr{I}$ ), and correspondingly the way in which the physical metric $\tilde{g}_{a b}$ is written as $\Omega^{-2} g_{a b}$ in terms of an auxilary unphysical metric. In other words, if $k$ is a smooth function defined in a neighborhood of the boundary of $\bar{M}$ such that $k \neq 0$ at null infinity, then our definition of an asymptotically flat metric is unchanged if we change the conformal factor to $\Omega^{\prime}=k \Omega$, the background metric to $\bar{g}^{\prime}{ }_{a b}=k^{2} \bar{g}_{a b}$, and the unphysical metric to $g_{a b}^{\prime}=k^{2} g_{a b}$.

As in 4 spacetime dimensions, the notion of weak asymptotic simplicity can be strengthened by requiring in addition that every inextendible null geodesic in $\left(\tilde{M}, \tilde{g}_{a b}\right)$ has precisely two endpoints on $\mathscr{I}$. Such a spacetime is then simply called asymptotically simple. This additional condition, combined with the fact that $\mathscr{I}$ is null, makes it possible to divide $\mathscr{I}$ into disjoint sets, $\mathscr{I}^{+}$and $\mathscr{I}^{-}$, on which future respectively past directed null geodesics have their endpoints. These sets are referred to as future respectively past null infinity. This condition also implies that $\left(\tilde{M}, \tilde{g}_{a b}\right)$ necessarily has to be globally hyperbolic, by a straightforward generalization of Prop. 6.9.2 of [17] to $d$ dimensions $^{7}$.

Item (1) of our definition is essentially the statement that, as manifold, $M$ looks near $\mathscr{I}$ like the background manifold $\bar{M}$ looks near its null boundary. Item (2) of the definition involves three different metrics: The physical $\tilde{g}_{a b}$, unphysical $g_{a b}$, and the background

\footnotetext{
${ }^{7}$ We also note that, by a straightforward generalization of Prop. 6.9.4 of 17 to $d$ dimensions, the additional condition is in fact only consistent with $\mathscr{I}$ having topology $\mathbb{R} \times S^{d-2}$. This agrees with the topology of the boundary of our background geometry.
} 
metric $\bar{g}_{a b}$. The physical and unphysical metric are related by the conformal factor, $\Omega$, which makes the unphysical metric smooth and at the same time brings null infinity, $\mathscr{I}$, to a "finite location", and the background metric is likewise related to Minkowski spacetime. The relation between the unphysical and background metric is given by the above set of equations in item (2), which specify the precise manner in which the unphysical metric approaches the background metric, and thus the precise sense in which our spacetime is required to flatten out at null infinity. Since $n_{a}$ is null relative to the background metric, it is also null relative to the metric $g_{a b}$, showing that $\mathscr{I}$ is a null surface in the unphysical spacetime $\left(M, g_{a b}\right)$. Since $\Omega^{2}$ times Minkowski spacetime is isometric to $\left(\bar{M}, \bar{g}_{a b}\right)$, our definition of asymptotic flatness trivially covers Minkowski spacetime.

Since we will be working with different metrics in this paper - physical and unphysicalit is necessary to specify a rule for raising and lowering indices of tensors. Our rule is that indices on tensor fields on $M$ without a "tilde" are raised and lowered with the unphysical metric, $g_{a b}$ and its inverse, whereas indices on tensor fields on $\tilde{M}$ with a "tilde" are raised and lowered with the physical metric, $\tilde{g}_{a b}$, and its inverse ${ }^{8}$.

Let us compare the above definition of asymptotic flatness with the behavior of the $d$-dimensional analog of the Schwarzschild metric, given by the line element

$$
\mathrm{d} \tilde{s}^{2}=-\left(1-c r^{-(d-3)}\right) \mathrm{d} t^{2}+\left(1-c r^{-(d-3)}\right)^{-1} \mathrm{~d} r^{2}+r^{2} \mathrm{~d} \sigma^{2}, \quad c>0,
$$

where $\mathrm{d} \sigma^{2}$ is the line element of a round $(d-2)$-dimensional sphere. Introducing a coordinate $u$ by the relation $\mathrm{d} u=\mathrm{d} t-\left(1-c r^{-(d-3)}\right)^{-1} \mathrm{~d} r$, the line element takes the form

$$
\mathrm{d} \tilde{s}^{2}=-2 \mathrm{~d} u \mathrm{~d} r-\mathrm{d} u^{2}+r^{2} \mathrm{~d} \sigma^{2}+c r^{-(d-3)} \mathrm{d} u^{2},
$$

where the first three terms on the right side are recognized as the Minkowski line element. Multiplying by our conformal factor $\Omega^{2}$, using $r^{-1}=O(\Omega)$, and using that $\Omega^{2}$ times the Minkowski metric is equal to our background metric $\mathrm{d} \bar{s}^{2}$ by construction, it follows that the unphysical Schwarzschild metric can be written as $\mathrm{d} s^{2}=\mathrm{d} \bar{s}^{2}+O\left(\Omega^{d-1}\right) \mathrm{d} u^{2}$ (noting that $u$ is a good coordinate at infinity). It follows that Schwarzschild spacetime is asymptotically flat in the sense of our definition, but it becomes flat at null infinity at a faster rate than that specified above in eqs. (3) and (4) in $d>4$. (In $d=4$, the relevant components drop off at the same rate, as specified in eqs. (3) and (4).)

The above definition of asymptotic flatness in even dimensions $d>4$ is not appropriate in odd spacetime dimension, since condition (3) in item 2 now says that the unphysical metric $g_{a b}$ differs from the smooth background metric $\bar{g}_{a b}$ by a half odd integer power of $\Omega$, and thereby manifestly contradicts the assumption in item 1 that $g_{a b}$ is smooth at the boundary. The powers of $\Omega$ appearing in eqs. (3) and (4) reflect the drop off behaviour of a linearized perturbation (see section 3), and it is hard to see how these powers could

\footnotetext{
${ }^{8}$ Note that this rule is consistent with our notation $g^{a b}$ and $\tilde{g}^{a b}$ for the inverse of the metrics $g_{a b}$ and $\tilde{g}_{a b}$.
} 
be essentially different from the ones in the full nonlinear theory. It therefore appears that the unphysical metric is generically at most $(d-3) / 2$ times differentiable at the boundary in odd dimensions. We note that it is also inconsistent in odd dimensions to postulate that the quantity $\Omega^{-(d-2) / 2}\left(g_{a b}-\bar{g}_{a b}\right)$ is smooth at the boundary as we did above in eq. (3) of item 2 in the even dimensional case, because the unphysical Schwarzschild metric $g_{a b}$ differs from the background $\bar{g}_{a b}$ by terms of order $\Omega^{d-1}$, i.e., by an even power of $\Omega$. Therefore, eq. (3) is definitely false for the Schwarzschild metric in odd dimensions. For the Schwarzschild metric, $\Omega^{-(d-1)}\left(g_{a b}-\bar{g}_{a b}\right)$ is smooth at the boundary (in even and odd dimensions), so one might be tempted to try this condition, together with suitable other conditions, as the definition of asymptotic flatness. However, this would eliminate from consideration all radiating spacetimes and is therefore not acceptable. One may try to bypass these problems by requiring appropriate lower differentiability properties of the corresponding quantities, but these seem neither to lead to a definition of asymptotic flatness that is stable under perturbations, as we briefly discuss in section 3, nor do those weaker conditions seem to be able to guarantee the existence of conserved quantities such as Bondi energy. Thus, it seems that a sensible definition of asymptotic simplicity at conformal infinity in odd spacetime dimensions would have to differ substantially from the one given above for even dimensions, and it is doubtful that such a definition can be cast into the framework of conformal infinity. For the rest of this paper, we will restrict attention to even spacetime dimensions.

We finally comment on how the above definition of asymptotic flatness in even spacetime dimensions $d>4$ compares to the usual definition [10] in 4 dimensions. In this definition, one simply demands that there exists some conformal factor, $\Omega$, such that the corresponding unphysical metric is smooth at $\mathscr{I}$ and such that $n_{a}$ is non-vanishing and null there ${ }^{9}$. This definition is different in appearance from that given above and avoids in particular the introduction of a background geometry. Nevertheless, the definition of asymptotic flatness in $d=4$ as just stated can be brought ${ }^{10}$ into a form that is very similar (but not identical) to the one given above for $d>4$. To see this in more detail, we recall that the usual definition of asymptotic flatness in 4 dimensions is equivalent 18 to the statement that the physical metric can be cast into "Bondi form"11 (see eqs.(14) and (31)-(34) of [2]),

$$
\begin{aligned}
\mathrm{d} \tilde{s}^{2}= & -2 \mathrm{~d} u \mathrm{~d} r-\mathrm{d} u^{2}+r^{2} \mathrm{~d} \sigma^{2} \\
& +O(r) \mathrm{d}(\text { angles })^{2}+O(1) \mathrm{d} u \mathrm{~d}(\text { angles })+O\left(r^{-1}\right) \mathrm{d} u^{2}+O\left(r^{-2}\right) \mathrm{d} u \mathrm{~d} r
\end{aligned}
$$

\footnotetext{
${ }^{9}$ The nullness of $n_{a}$ follows from the first condition if Einstein's equations with vanishing stress energy at null infinity are assumed.

${ }^{10}$ We emphasize, however, that an analogous statement is not true in $d>4$. Namely, it is not true that our definition of asymptotic flatness in higher dimensions is equivalent to the statement that there exists some conformal factor, $\Omega$, such that the corresponding unphysical metric is smooth at $\mathscr{I}$ and such that $n_{a}$ is non-vanishing and null there.

11 It is assumed in the derivation of eq. (7) that the vacuum Einstein equations are satisfied.
} 
in suitable coordinates near null infinity, where the first line is recognized as the Minkowski line element, and where "angles" stands for the usual polar angles of $S^{2}$. In $d>4$ spacetime dimensions our asymptotic flatness conditions eqs. (3) and (44) in effect state that the physical line element can be written in the form

$$
\begin{aligned}
\mathrm{d} \tilde{s}^{2}= & -2 \mathrm{~d} u \mathrm{~d} r-\mathrm{d} u^{2}+r^{2} \mathrm{~d} \sigma^{2} \\
& +O\left(r^{-\frac{d-4}{2}}\right) \mathrm{d}(\text { angles })^{2}+O\left(r^{-\frac{d-4}{2}}\right) \mathrm{d} u \mathrm{~d}(\text { angles }) \\
& +O\left(r^{-\frac{d-2}{2}}\right) \mathrm{d} u^{2}+O\left(r^{-\frac{d}{2}}\right) \mathrm{d} u \mathrm{~d} r,
\end{aligned}
$$

where "angles" now stands for the polar angles of $S^{d-2}$. One notices that the Bondi form (8) in $d>4$ does not reduce to eq. (7) when $d$ is set to 4 . The difference between the two expression arises from the $\mathrm{d}$ (angles) ${ }^{2}$-term, which quantifies the perturbations in the size of the cross sections of a lightcone relative to Minkowski spacetime. According to eq. (7), this term is of order $O(1)$ in $d=4$ for a radiating metric, whereas eq. (8) would say that it ought to be of order $O\left(r^{-1}\right)$. The latter is simply wrong for a radiating metric in 4 dimensions. This difference can be traced back to the last of conditions (3) in $d>4$ dimensions, which therefore does not hold in $d=4$. This special feature of 4 dimensions will be reflected in corresponding differences in our discussion of the Bondi energy in dimensions $d>4$. We will therefore, for the rest of this paper, keep the case $d=4$ separate and assume throughout that $d>4$ (and even). Our formulas will not be valid in $d=4$ unless stated otherwise.

A diffeomorphism $\phi$ such that $\phi^{*} \tilde{g}_{a b}$ is asymtpotically flat whenever $\tilde{g}_{a b}$ is asymptotically flat is called an asymptotic symmetry. It is clear that the asymptotic symmetries form a group under the composition of two diffeomorphisms. Clearly, the property of being an asymptotic symmetry is only related to the behavior of $\phi$ near the boundary. An infinitesimal asymptotic symmetry is a smooth vector field $\xi^{a}$ on $\tilde{M}$ that has a smooth extension (denoted by the same symbol) to the unphysical manifold, $M$, and which generates a 1-parameter group of asymptotic symmetries. It is a direct consequence of our definitions that the quantity

$$
\chi_{a b}=\Omega^{-\frac{d-6}{2}} £_{\xi} \tilde{g}_{a b}=2 \Omega^{-\frac{d-2}{2}}\left(\nabla_{(a} \xi_{b)}-\Omega^{-1} n^{c} \xi_{c} g_{a b}\right)
$$

then has to satisfy

$$
\chi_{a b}=O(1), \quad \chi_{a}{ }^{a}=O(\Omega), \quad \chi_{a b} n^{a}=O(\Omega), \quad \chi_{a b} n^{a} n^{b}=O\left(\Omega^{2}\right), \quad \xi^{a} n_{a}=O(\Omega),
$$

where here and in the following we are using the abbreviation

$$
n_{a}=\nabla_{a} \Omega .
$$

Conversely, if the above relations are satisfied for some asymptotically flat spacetime, then $\xi^{a}$ is an infinitesimal asymptotic symmetry. The classification of asymptotic symmetries in $d>4$ differs from that in 4 dimensions. We will discuss this issue in some detail below in section 5 and in appendix $\mathrm{C}$, as well as in a fourthcoming paper [19. 


\section{$3 \quad$ Stability of asymptotic flatness to linear order}

In this section we justify the definition of asymptotic flatness for even $d$ given in the previous section by showing that it is stable under linear perturbations. What we mean by this is the following. Suppose that $\left(\tilde{M}, \tilde{g}_{a b}\right)$ is an asymptotically flat spacetime that is also a solution to the vacuum Einstein equation, $\tilde{R}_{a b}=0$. Consider a solution, $\delta \tilde{g}_{a b}$, to the linearized equations of motion around this background ${ }^{12}$,

$$
0=\delta \tilde{R}_{a b}=\frac{1}{2}\left(-\tilde{\nabla}^{m} \tilde{\nabla}_{m} \delta \tilde{g}_{a b}-\tilde{\nabla}_{a} \tilde{\nabla}_{b} \delta \tilde{g}_{m}{ }^{m}+2 \tilde{\nabla}^{m} \tilde{\nabla}_{(a} \delta \tilde{g}_{b) m}\right),
$$

which has the property that the restriction of $\delta \tilde{g}_{a b}$ to a Cauchy surface has compact support. We will show in this section that there exists a gauge (the transverse traceless gauge works) such that, setting $\delta g_{a b}=\Omega^{2} \delta \tilde{g}_{a b}$,

$$
\delta g_{a b}=O\left(\Omega^{\frac{d-2}{2}}\right), \quad \delta g_{a b} n^{a}=O\left(\Omega^{\frac{d}{2}}\right), \quad \delta g_{a b} n^{a} n^{b}=O\left(\Omega^{\frac{d+2}{2}}\right), \quad g^{a b} \delta g_{a b}=O\left(\Omega^{\frac{d}{2}}\right),
$$

at $\mathscr{I}$ for all even $d>4$. These conditions are recognized as the linearized version of our definition of asymptotic flatness, eqs. (3) and (41), about an asymptotically flat background. Our definition of asymptotic flatness is therefore stable to linear order.

We have emphasized above that our decay properties of the metric perturbations are valid only in a particular gauge. Indeed, since the linearized equations of motion, $\delta \tilde{R}_{a b}=0$, are invariant under a gauge transformation $\delta \tilde{g}_{a b} \rightarrow \delta \tilde{g}_{a b}+£_{\xi} \tilde{g}_{a b}$ with $\xi^{a}$ an arbitrary smooth vector field on $\tilde{M}$, eq. (13) cannot possibly be true in an arbitrary gauge. For if it were to hold in one gauge, it would certainly not hold in a gauge with a $\xi^{a}$ that is very badly behaved at $\mathscr{I}$. Thus, the specification of an admissible gauge choice for the metric perturbation is an important part of the demonstration of eq. (13).

A proof that asymptotic flatness is stable to linear order in 4 dimensions was given by Geroch and Xanthopoulos [12. Their argument consists of the following two steps: One first writes the linearized equations of motions (12) in terms of the unphysical metric and derivative operator and introduces new field variables such that eq. (12) is transformed into a hyperbolic system of partial differential equations whose coefficients are either manifestly regular functions as one approaches $\mathscr{I}$ or can be made to vanish by a suitable gauge choice. One then argues, using standard existence and uniqueness results for solutions of hyperbolic partial differential equations, that the new variables therefore have a smooth extension to the unphysical spacetime. (Here one needs to use that the perturbation has compactly supported initial data.) Translating this statement about the new variables back into a statement about the metric perturbation, one finds the decay properties of the metric perturbation at $\mathscr{I}$.

\footnotetext{
${ }^{12}$ In this section, by "background" we mean the "unperturbed" physical spacetime $\left(\tilde{M}, \tilde{g}_{a b}\right)$, unless otherwise stated. This should not be confused with the background (2), which is our reference spacetime for defining asymptotic flatness.
} 
We here use this basic strategy to analyze the decay at $\mathscr{I}$ of metric perturbations in even dimensions $d>4$. The second step does not depend on the dimension of the spacetime, since it only involves general properties of hyperbolic differential equations. By contrast, the first step, i.e. the actual choice of variables and gauge conditions, is different in nature in $d>4$ dimensions as compared to $d=4$. Finding the appropriate variables and gauge conditions that do the job is, of course, the hard part of the analysis. It needs to be done before writing down any decay properties of the perturbations, which are then supposed to follow from the precise form of the new variables and gauge conditions. We here present things in the opposite order in order to simplify the exposition. (Throughout the rest of this section, $d>4$ and even will be assumed.)

Concerning the proper choice of gauge, we consider the 1-form

$$
\tilde{v}_{a}=\tilde{g}^{b c} \tilde{\nabla}_{c} \delta \tilde{g}_{a b}-\tilde{g}^{b c} \tilde{\nabla}_{a} \delta \tilde{g}_{b c},
$$

which is equal to (dual of) the integrand of the surface term arising when varying the Einstein-Hilbert action. Under a gauge transformation, $\delta \tilde{g}_{a b} \rightarrow \delta \tilde{g}_{a b}+£_{\xi} \tilde{g}_{a b}$, this quantity is seen to transform as

$$
\tilde{v}^{a} \rightarrow \tilde{v}^{a}+2 \tilde{\nabla}_{b} \tilde{\nabla}^{[b} \xi^{a]}
$$

where the vacuum Einstein equations $\tilde{R}_{a b}=0$ have been used. On the other hand, the variation of the Ricci tensor can be seen to satisfy

$$
\tilde{g}^{a b} \delta \tilde{R}_{a b}=\tilde{\nabla}^{a} \tilde{v}_{a}
$$

so that we have $\tilde{\nabla}^{a} \tilde{v}_{a}=0$ when the linearized Einstein equations hold. Thus, by eq. (15), we can set $\tilde{v}_{a}=0$ throughout $\tilde{M}$ by a choice of gauge transformation when the linearized Einstein equations hold ${ }^{13}$. This gauge choice is invariant under further gauge transformations of the form $\delta \tilde{g}_{a b} \rightarrow \delta \tilde{g}_{a b}+£_{\xi} \tilde{g}_{a b}$, with $\xi^{a}=\tilde{\nabla}^{a} \xi$ and $\xi$ any smooth function on $\tilde{M}$, which can be used, for example, to impose a gauge condition on the trace of the perturbation. We impose

$$
\delta \tilde{g}_{a}^{a}=0,
$$

which can always be realized since the trace transforms as

$$
\delta \tilde{g}_{a}^{a} \rightarrow \delta \tilde{g}_{a}^{a}+\tilde{\nabla}^{a} \tilde{\nabla}_{a} \xi
$$

under the remaining gauge transformations. Thus, our gauge conditions are eq. (17) and $\tilde{v}_{a}=0$. Together, they are equivalent to the transverse traceless gauge condition, $\tilde{\nabla}^{a} \delta \tilde{g}_{a b}=\delta \tilde{g}_{m}{ }^{m}=0$.

Concerning the proper choice of field variables when $d>4$, we consider

$$
\tau_{a b} \equiv \Omega^{-\frac{d-2}{2}} \delta g_{a b}, \quad \tau_{a} \equiv \Omega^{-1} \tau_{a b} n^{b}, \quad u \equiv \nabla^{a} \tau_{a} .
$$

\footnotetext{
${ }^{13}$ Note that $0=\tilde{v}^{a}+2 \tilde{\nabla}_{b} \tilde{\nabla}^{[b} \xi^{a]}$ has the same form as Maxwell's equation for a vector potential, with a divergence free source. A solution therefore exists by the same arguments as for Maxwell's equations.
} 
We substitute the definitions (19) into the linearized Einstein equation, and use the background Einstein equation, $\tilde{R}_{a b}=0$, as well as the well-known relations between the physical and unphysical derivative operator and Ricci tensor,

$$
\begin{gathered}
\tilde{\nabla}_{a} k_{b}=\nabla_{a} k_{b}+\Omega^{-1}\left(2 \delta^{c}{ }_{(a} n_{b)}-g_{a b} n^{c}\right) k_{c} \\
\tilde{R}_{a b}=R_{a b}+\Omega^{-1}\left[(d-2) \nabla_{a} n_{b}+\left(\nabla_{m} n^{m}\right) g_{a b}-(d-1) f g_{a b}\right] .
\end{gathered}
$$

A lengthy calculation shows that the result can be written in the form

$$
\begin{aligned}
0=2 \Omega^{-\frac{d-2}{2}} \delta \tilde{R}_{a b}= & -\nabla^{c} \nabla_{c} \tau_{a b}+\nabla_{a} \nabla_{b} \tau+4 \nabla_{(a} \tau_{b)}+2 \nabla_{(a} y_{b)}-2 u g_{a b} \\
& -2 R_{a c b d} \tau^{c d}-\frac{(d-6)}{2(d-2)} R_{a b} \tau+\frac{(d-2)}{4(d-1)} R \tau_{a b}+\frac{(d-6)}{4(d-1)(d-2)} g_{a b} R \tau \\
& +\Omega^{-1}(d-2) n_{(a} y_{b)}+\Omega^{-1} g_{a b}\left((d-2) n_{c} \tau^{c}+n^{c} \nabla_{c} \tau+\frac{(3 d-10)}{4} f \tau\right) \\
& +(d-4) \Omega^{-1} n_{(a} \nabla_{b)} \tau+\frac{(d-6)(d-4)}{4} \Omega^{-2} n_{a} n_{b} \tau,
\end{aligned}
$$

where we have used the shorthand notation

$$
\tau=\tau_{a}^{a}, \quad y_{a}=\Omega^{-\frac{d-2}{2}} \tilde{v}_{a}=\nabla^{c} \tau_{c a}-\nabla_{a} \tau-\frac{(d+2)}{2} \tau_{a}-\frac{(d-4)}{2} \Omega^{-1} n_{a} \tau,
$$

as well as

$$
f=\Omega^{-1} n_{a} n^{a}
$$

We now substitute our gauge conditions, $y_{a}=\tau=0$, using in particular that

$$
0=n^{a} y_{a}=\frac{1}{2}\left(u+\frac{1}{(d-2)} R^{a b} \tau_{a b}\right)-\frac{d}{4} \Omega^{-1} n^{a} \tau_{a}
$$

which follows by combining our gauge condition with the background Einstein equation, to get rid of the $\Omega^{-1} n^{a} \tau_{a}$ term in eq. (22). Then eq. (22) reduces to

$$
\nabla^{c} \nabla_{c} \tau_{a b}=4 \nabla_{(a} \tau_{b)}-\frac{4}{d} u g_{a b}+\frac{(d-2)}{4(d-1)} R \tau_{a b}+\frac{2}{d} R^{c d} \tau_{c d} g_{a b}-2 R_{a c b d} \tau^{c d}
$$

where all singular terms now have dropped out due to our choice of variables and gauge condition. We are, however, not done yet since we also need appropriate equations for the remaining variables, $\tau_{a}, u$.

In order to get an equation for $\tau_{a}$, we take the divergence of eq. (26) with respect to the unphysical metric and use again the transverse traceless gauge condition, $y_{a}=\tau=0$. 
This gives

$$
\begin{aligned}
(d-2) \nabla^{c} \nabla_{c} \tau_{a}= & \frac{4(d-2)}{d} \nabla_{a} u+\frac{4}{d} R^{b c} \nabla_{a} \tau_{b c}-2 R^{b c} \nabla_{b} \tau_{c a} \\
& +\left(\frac{(d+2)(d-2)}{4(d-1)} R g_{a b}+4 R_{a b}\right) \tau^{b} \\
& +2\left(\nabla_{b} R_{c a}-\frac{(d-2)}{d} \nabla_{a} R_{b c}-\frac{d}{4(d-1)}\left(\nabla_{c} R\right) g_{a b}\right) \tau^{b c}
\end{aligned}
$$

Finally, in order to get an equation for $u$, we take a further divergence of eq. (27). We use eq. (26) and the divergence of the transverse traceless gauge condition $\nabla^{a} y_{a}=\tau=0$ to eliminate second derivatives of $\tau_{a b}$, giving

$$
\begin{aligned}
&(d-2)(d-4) \nabla^{c} \nabla_{c} u=-2(d+2)(d-4) R_{a b} \nabla^{a} \tau^{b}-2(d-4) \nabla^{a} R^{b c} \nabla_{a} \tau_{b c} \\
&+\frac{(d-4)\left(d^{3}+4 d^{2}+12 d-16\right)}{4 d(d-1)} R u-\frac{d(d-2)(d-4)}{2(d-1)}\left(\nabla_{c} R\right) \tau^{c} \\
&+\left(\frac{d(d-2)}{2(d-1)} \nabla_{a} \nabla_{b} R-2(d-2) \nabla^{c} \nabla_{c} R_{a b}\right. \\
&\left.-8 R^{c d} R_{a c b d}+\frac{d^{2}+6 d-8}{d(d-1)} R R_{a b}\right) \tau^{a b} .
\end{aligned}
$$

Equations (26), (27) and (28) form a system of linear partial differential equations for the variables $\tau_{a b}, \tau_{a}, u$ in the unphysical spacetime $M$, with coefficients that are given in terms of the unphysical Riemann and Ricci tensor and its first and second derivatives. No terms containing explicitly inverse powers of $\Omega$ appear due to our particular choice of variables and gauge conditions. Introducing the shorthand notation $\phi_{\alpha}=\left(\tau_{a b}, \tau_{a}, u\right)$, this system can be rewritten more compactly as

$$
g^{a b} \nabla_{a} \nabla_{b} \phi_{\alpha}=A_{\alpha}^{\beta a} \nabla_{a} \phi_{\beta}+B_{\alpha}^{\beta} \phi_{\beta}
$$

It follows from our definition of an asymptotically flat spacetime that the coefficients $A_{\alpha}{ }^{\beta a}, B_{\alpha}{ }^{\beta}$ in this system are smooth tensor fields up to and on the boundary. Equation (29) therefore forms a hyperbolic system ${ }^{14}$ of partial differential equations with coefficients that are smooth functions up to and on $\mathscr{I}$. Hence, this system possesses a well-posed initial value formulation [17] in the unphysical spacetime. If $\phi_{\alpha}$ has compactly supported initial data as we have assumed, then we conclude by the general argument given in [12] that $\phi_{\alpha}$ and hence $\tau_{a b}, \tau_{a}$ and $u$, extend to smooth tensor fields at $\mathscr{I}$. In

\footnotetext{
${ }^{14}$ I.e., roughly speaking, does not contain any second derivatives other than the wave operator. Such terms could have arisen via expressions such as $R^{a b c d} \nabla_{a} \nabla_{c} \tau_{b d}$ which, as we note, could not be eliminated in favor of first derivative terms via our gauge condition, $\nabla^{m} y_{m}=0$. Fortunately, these terms happen to cancel.
} 
combination with eq. (25), this implies moreover that $\Omega^{-1} n^{a} \tau_{a}$ is smooth at $\mathscr{I}$ in the transverse traceless gauge, and hence that $n^{a} \tau_{a}=O(\Omega)$. Substituting back the definition (19) of $\tau_{a b}$ and $\tau_{a}$ and the gauge condition $\tau=0$ in terms of $\delta g_{a b}$, we altogether find that the desired drop off properties (13) hold for the linearized perturbation at $\mathscr{I}$. Thus, we have shown that our definition of asymptotic simplicity given in the previous section is stable under linear perturbations when $d>4$ and even.

For completeness, we now comment upon the status of the above argument in the case when $d>4$ and odd. In that case, the algebra leading to eq. (29) is identical as in the case $d>4$ and even, but the coefficients $g^{a b}, A_{\alpha}{ }^{\beta a}, B_{\alpha}{ }^{\beta}$ in this system now cannot be assumed to be smooth at the boundary, since the unphysical metric $g_{a b}$ itself does not have this property (see the discussion in section 2). Instead, since $g_{a b}$ can at best be expected to be of differentiability class $C^{s}, s=(d-2) / 2$ at the boundary, we can at best expect that $A_{\alpha}{ }^{\beta a} \in C^{s-3}, B_{\alpha}{ }^{\beta} \in C^{s-4}$ at the boundary. On the other hand, the standard existence and uniqueness results for linear hyperbolic equations of the form (29) require a higher degree of regularity ${ }^{15}$ for the coefficients and therefore do not guarantee the existence of a solution to (29). Thus, unlike in the case of even $d>4$, we now cannot conclude that $\phi_{\alpha}$ and hence $\tau_{a b}, \tau_{a}$ and $u$, extend to, say continuous, tensor fields at $\mathscr{I}$, and we therefore also cannot conclude that e.g. $\delta g_{a b}$ is given by $\Omega^{(d-2) / 2}$ times a continuous function. Thus, our stability proof breaks down in odd dimensions. We believe that this is an indication that a geometric definition of asymptotic simplicity that is stable against perturbations is not possible in odd dimensions.

In $d=4$ spacetime dimensions, the above system of equations for $\tau_{a b}, \tau_{a}, u$ fails to be even hyperbolic, since the "box term" drops out in the equation (28) for $u$. Thus, the above choice of variables and gauge does not work in $d=4$. A set of variables and gauge conditions that works in 4 dimensions has been found by Geroch and Xanthopoulos [12] These variables are $\tau_{a b}, \tau_{b}$ and $\sigma=\Omega^{-1}\left(n^{a} \nabla_{a} \tau+\frac{1}{2} n^{a} \tau_{a}+\frac{1}{4} f \tau\right)$. The gauge condition is chosen to be $y_{a}=0$, together with a certain complicated gauge condition on the trace of the perturbation instead of $\tau=0$. With this choice of variables and gauge conditions in place, it is then shown that $\tau_{a b}, \tau_{b}, \sigma$ satisfy a system of hyperbolic equations with coefficients that are smooth at $\mathscr{I}$ (assuming that the unphysical metric is smooth at $\mathscr{I}$ ). It follows now that the metric perturbation has the fall-off rate

$$
\delta g_{a b}=O(\Omega), \quad \delta g_{a b} n^{b}=O\left(\Omega^{2}\right), \quad \delta g_{a b} n^{a} n^{b}=O\left(\Omega^{3}\right)
$$

in this gauge. This differs from the corresponding result eq. (13) $d>4$ in that the trace of the perturbation is now only falling off as fast as the metric perturbation itself, $\delta g_{m}{ }^{m}=O(\Omega)$, rather than one power faster as in $d>4$. This confirms the observation

\footnotetext{
${ }^{15}$ In order to guarantee existence and uniqueness of a solution in the class $\phi_{\alpha} \in W^{d / 2+2+A}, A \geq 0$ (we mean the Sobolev space), one needs $g_{a b} \in W^{d / 2+2+A}, A_{\alpha}{ }^{\beta a} \in W^{d / 2+1+A}$ and $B_{\alpha}{ }^{\beta} \in W^{d / 2+1+A}$. This is stronger than what we know.
} 
already made in the previous section that we cannot impose the second of eqs. (41) in 4 dimensions, which, as we note, would be the non-linear analog of the condition $\delta g_{m}{ }^{m}=$ $O\left(\Omega^{2}\right)$. Hence, it is seen that the definition of asymptotic flatness is qualitatively different in $d>4$ dimensions. As we will see, this has consequences for our analysis of the Bondi energy in $d>4$ dimensions.

\section{General strategy for defining "conserved" quanti- ties at infinity}

In this section, we review the general algorithm given by Wald and Zoupas [14 for defining "charges" associated with symmetries preserving a given set of "boundary conditions" in the context of theories derived from a diffeomorphism covariant Lagrangian. This will later be used to define the Bondi energy in $d$-dimensional general relativity as the generator conjugate to an appropriately defined asymptotic time translation symmetry.

The algorithm [14] applies to arbitrary theories derived from a diffeomorphism covariant Lagrangian. We will focus here on vacuum general relativity in $d$ dimensions, defined by the Lagrangian density (viewed as a $d$-form)

$$
L=\frac{1}{16 \pi G} \tilde{R} \tilde{\epsilon}
$$

and the boundary conditions specified in our definition of asymptotic flatness.

One considers the variation of $L$, which can always be written in the form

$$
\delta L=E+\mathrm{d} \theta
$$

where $E$ are the equations of motion; in our case

$$
E_{a_{1} \ldots a_{d}}=\frac{1}{16 \pi G}\left(\tilde{R}_{b c}-\frac{1}{2} \tilde{R} \tilde{g}_{b c}\right) \delta \tilde{g}^{b c} \tilde{\epsilon}_{a_{1} \ldots a_{d}}
$$

and where $\mathrm{d} \theta$ is the exterior differential of a $(d-1)$-form $\theta$, given in our case by

$$
\theta_{a_{1} \ldots a_{d-1}}=\frac{1}{16 \pi G} \tilde{v}^{c} \tilde{\epsilon}_{c a_{1} \ldots a_{d-1}}
$$

where $\tilde{v}_{a}$ is given in terms of $\delta \tilde{g}_{a b}$ by eq. (14). The antisymmetrized second variation ${ }^{16} \omega$ of $\theta$ defines the (dualized) symplectic current,

$$
\omega\left(\tilde{g} ; \delta_{1} \tilde{g}, \delta_{2} \tilde{g}\right)=\delta_{1} \theta\left(\tilde{g} ; \delta_{2} \tilde{g}\right)-\delta_{2} \theta\left(\tilde{g} ; \delta_{1} \tilde{g}\right),
$$

\footnotetext{
${ }^{16}$ Here, and in similar other formulas involving second variations, we assume without loss of generality that the variations commute, i.e., that $\delta_{1}\left(\delta_{2} \tilde{g}\right)-\delta_{2}\left(\delta_{1} \tilde{g}\right)=0$.
} 
so that $\omega$ depends on the unperturbed metric and is skew in the pair of perturbations $\left(\delta_{1} \tilde{g}_{a b}, \delta_{2} \tilde{g}_{a b}\right)$. It is given in our case by

$$
\omega_{a_{1} \ldots a_{d-1}}=\frac{1}{16 \pi G} \tilde{w}^{c} \tilde{\epsilon}_{c a_{1} \ldots a_{d-1}}
$$

where $\tilde{w}^{c}$ is the symplectic current vector

$$
\tilde{w}^{a}=\tilde{P}^{a b c d e f}\left(\delta_{1} \tilde{g}_{b c} \tilde{\nabla}_{d} \delta_{2} \tilde{g}_{e f}-\delta_{2} \tilde{g}_{b c} \tilde{\nabla}_{d} \delta_{1} \tilde{g}_{e f}\right)
$$

with

$$
\tilde{P}^{a b c d e f}=\tilde{g}^{a e} \tilde{g}^{f b} \tilde{g}^{c d}-\frac{1}{2} \tilde{g}^{a d} \tilde{g}^{b e} \tilde{g}^{f c}-\frac{1}{2} \tilde{g}^{a b} \tilde{g}^{c d} \tilde{g}^{e f}-\frac{1}{2} \tilde{g}^{b c} \tilde{g}^{a e} \tilde{g}^{f d}+\frac{1}{2} \tilde{g}^{b c} \tilde{g}^{a d} \tilde{g}^{e f} .
$$

The integral of the symplectic current over an achronal $(d-1)$-dimensional submanifold $\tilde{\Sigma}$ of $\tilde{M}$ defines the symplectic structure, $\sigma$, of general relativity

$$
\sigma\left(\tilde{g} ; \delta_{1} \tilde{g}, \delta_{2} \tilde{g}\right)=\int_{\tilde{\Sigma}} \omega .
$$

It can be shown that, when both $\delta_{1} \tilde{g}_{a b}$ and $\delta_{2} \tilde{g}_{a b}$ satisfy the linearized equations of motion (12), then $\mathrm{d} \omega=0$, or, what is the same thing, that the symplectic current (37) is conserved, $\tilde{\nabla}^{a} \tilde{w}_{a}=0$. Consequently, the symplectic structure $\sigma$ does not depend on the choice of $\Sigma$, when $\delta_{1} \tilde{g}_{a b}$ and $\delta_{2} \tilde{g}_{a b}$ satisfy the linearized equations of motion with compactly supported initial data.

The algorithm 14 for defining generators associated with asymptotic symmetries now consists of the following steps. First, check whether the symplectic current form $\omega\left(\tilde{g} ; \delta_{1} \tilde{g}, \delta_{2} \tilde{g}\right)$ has a well-defined (i.e., finite) extension to $\mathscr{I}$ for all asymptotically flat metrics satisfying Einstein's equation and all metric perturbations preserving asymptotic flatness to first order, i.e., for all linear perturbations satisfying the linearized equations of motion and eqs. (13). If this is the case, one secondly seeks a $(d-1)$-form $\Theta(\tilde{g} ; \delta \tilde{g})$ on $\mathscr{I}$ which is linear in the perturbation, $\delta \tilde{g}_{a b}$, which is locally constructed out of the metric $\tilde{g}_{a b}$ and its derivatives at the boundary and any further quantities arising in the specification of the boundary condition, and which has the property that the pull back of the symplectic current $\omega$ to $\mathscr{I}$ can be written as the antisymmetrized variation of $\Theta$,

$$
\omega\left(\tilde{g} ; \delta_{1} \tilde{g}, \delta_{2} \tilde{g}\right)=\delta_{1} \Theta\left(\tilde{g} ; \delta_{2} \tilde{g}\right)-\delta_{2} \Theta\left(\tilde{g} ; \delta_{1} \tilde{g}\right) .
$$

If such a symplectic potential $\Theta$ exists (which is by no means guaranteed and depends crucially on the precise form of the boundary conditions under consideration), then a generator conjugate to an asymptotic symmetry can be defined as follows: If $\xi^{a}$ is a vector field on $\tilde{M}$ representing an infinitesimal asymptotic symmetry, define the associated charge $\mathcal{H}_{\xi}$ by the formula

$$
\delta \mathcal{H}_{\xi}=\int_{B}\left(\delta Q_{\xi}-\xi \cdot \theta\right)+\int_{B} \xi \cdot \Theta
$$


where $B$ is the cross section of $\mathscr{I}$ at which the generator is to be evaluated and where $Q_{\xi}$ is the Noether-charge $(d-2)$-form, given in the present case by

$$
Q_{a_{1} \ldots a_{d-2}}=-\frac{1}{16 \pi G}\left(\tilde{\nabla}^{b} \xi^{c}\right) \tilde{\epsilon}_{b c a_{1} \ldots a_{d-2}} .
$$

In these formulae, the notation " $\xi \cdot A$ " means that the vector field $\xi^{a}$ is contracted into the first index of a differential form $A$.

It is not immediately evident from what we have said so far that eq. (41) actually defines a generator (up to an arbitrary constant), i.e., that the right side of eq. (41) is indeed the " $\delta$ " of some quantity $\mathcal{H}_{\xi}$. To see this, one first verifies that the right side of eq. (41) has a vanishing anti-symmetrized second variation ${ }^{17}$. This is certainly a necessary condition for it to arise as the " $\delta$ " of some quantity $\mathcal{H}_{\xi}$, for we always have $\left(\delta_{1} \delta_{2}-\delta_{2} \delta_{1}\right) \mathcal{H}_{\xi}=0$. As argued in [14], this is also a sufficient condition if one assumes that the space of asymptotically flat metrics is simply connected ${ }^{18}$. For the cases considered in this paper, we will prove existence of a $\mathcal{H}_{\xi}$ and provide an explicit expression solving eq. (41). The arbitrary constant is fixed by setting $\mathcal{H}_{\xi}$ equal to 0 on Minkowski spacetime.

The "flux" through a segment $\mathscr{S}$ of $\mathscr{I}$ bounded by two cross-sections $B_{1}$ and $B_{2}$ associated with the infinitesimal symmetry $\xi^{a}$ is defined to be the difference

$$
F_{\xi}=\mathcal{H}_{\xi}\left(B_{2}\right)-\mathcal{H}_{\xi}\left(B_{1}\right)
$$

One finds the simple formula 14

$$
F_{\xi}=\int_{\mathscr{S}} \Theta\left(\tilde{g} ; £_{\xi} \tilde{g}\right)
$$

We finally comment on the meaning of the integrals in eq. (41). The second integral on the right side of (41) has a straightforward meaning since it has been assumed that the integrand, $\Theta$, is well defined and smooth on $\mathscr{I}$. This is, however, not so for the first integral on the right side of (41), because the integrand is defined only in the interior of the spacetime. This integral is to be understood instead as the limit of the corresponding integrals for a sequence of closed, smooth $(d-2)$-surfaces $B_{i}$ in the interior of the physical spacetime $\tilde{M}$ that smoothly approach the cross section $B$ of $\mathscr{I}$ as $i \rightarrow \infty$. The following argument [14] shows that this limit indeed exists under the assumptions that have been made: Let $I_{i}=\int_{B_{i}}\left[\delta Q_{\xi}-\xi \cdot \theta\right]$. Then, since

$$
\mathrm{d}\left[\delta Q_{\xi}(\tilde{g}, \delta \tilde{g})-\xi \cdot \theta(\tilde{g} ; \delta \tilde{g})\right]=\omega\left(\tilde{g} ; \delta \tilde{g}, £_{\xi} \tilde{g}\right),
$$

we have, by Stoke's theorem

$$
I_{i}-I_{j}=\int_{\tilde{\Sigma}_{i j}} \omega\left(\tilde{g} ; \delta \tilde{g}, £_{\xi} \tilde{g}\right),
$$

${ }^{17}$ This would not be so if we had not added the $\Theta$-term to the expression for $\delta \mathcal{H}_{\xi}$ !

${ }^{18}$ Note the analogy to "Poincare's lemma" which says that every closed 1-form on a simply connected space is exact, i.e., the "d" of some scalar function. 
where $\tilde{\Sigma}_{i j}$ is a smooth spacelike $(d-1)$-surface bounded by $B_{i}$ and $B_{j}$. But $\omega$ has a smooth extension to $\mathscr{I}$ by assumption, so the right side of this equation goes to 0 as $i, j \rightarrow \infty$.

\section{The Bondi energy formula}

The aim of this section is to implement the strategy of the previous section for an asymptotic time translation symmetry in even spacetime dimensions $d>4$, i.e., to show that a generator $\mathcal{H}_{\xi}$ exists for such a symmetry, and to derive an expression for this generator. We will take this $\mathcal{H}_{\xi}$ as the definition of the Bondi energy-momentum (Bondi energy, for short) in spacetimes of even dimension $d>4$.

The crucial issue regarding the existence of a generator conjugate to asymptotic symmetries is whether the symplectic current $\omega$ has a (finite) restriction to $\mathscr{I}$ and whether there exists, under our choice of boundary conditions, a potential $\Theta$ for the pull-back (50) of the symplectic current density to $\mathscr{I}$, i.e., a $\Theta$ satisfying (40). We now examine these issues.

We fix the conformal factor $\Omega$ once and for all, so that, if $\left(\tilde{M}, \tilde{g}_{a b}\right)$ is an asymptotically flat spacetime, then $g_{a b}=\Omega^{2} \tilde{g}_{a b}$ satisfies eqs. (69) with this fixed choice of $\Omega$. Consider a solution $\delta \tilde{g}_{a b}$ of the linearized field equations that preserves asymptotic flatness to first order. Then the quantities $\tau_{a b}=\Omega^{-\frac{d-6}{2}} \delta \tilde{g}_{a b}, \tau_{a}=\Omega^{-1} \tau_{a b} n^{b}$ are finite and smooth at $\mathscr{I}$ and $\tau_{a}{ }^{a}$ and $n^{a} \tau_{a}$ vanish at $\mathscr{I}$. We substitute these relations into the definition of the symplectic current, use the relation (20) between the physical and unphysical derivative, the relation

$$
\tilde{\epsilon}_{s_{1} \ldots s_{d}}=\Omega^{-d} \epsilon_{s_{1} \ldots s_{d}}
$$

between the physical and unphysical volume element, and evaluate at $\mathscr{I}$. After some algebra, we find the simple result

$$
\omega_{s_{1} \ldots s_{d}}=\frac{1}{32 \pi G}\left(\tau_{1}^{b c} \nabla^{m} \tau_{2 b c}-\tau_{2}^{b c} \nabla^{m} \tau_{1 b c}\right) \epsilon_{m a_{1} \ldots a_{d-1}}+O(\Omega),
$$

noticing that this is finite at $\mathscr{I}$ (this formula is valid only in $d>4$ ). This expression can be rewritten somewhat more conveniently introducing a $(d-1)$-form ${ }^{(d-1)} \epsilon$ by the formula ${ }^{19}$

$$
{ }^{(d)} \epsilon_{m a_{1} \ldots a_{d-1}}=d \cdot n_{[m}{ }^{(d-1)} \epsilon_{\left.a_{1} \ldots a_{d-1}\right]}=\left(n \wedge^{(d-1)} \epsilon\right)_{m a_{1} \ldots a_{d-1}},
$$

where we have put a superscript on the quantities in order to indicate the degree of the form. The pull back to $\mathscr{I}$ of the symplectic current $(d-1)$-form $\omega$ can then be written as

$$
\zeta^{*} \omega_{a_{1} \ldots a_{d-1}}=\frac{1}{32 \pi G}\left(\tau_{1}^{b c} n^{m} \nabla_{m} \tau_{2 b c}-\tau_{2}^{b c} n^{m} \nabla_{m} \tau_{1 b c}\right) \epsilon_{a_{1} \ldots a_{d-1}},
$$

\footnotetext{
${ }^{19}$ Note that ${ }^{(d-1)} \epsilon$ is only defined up to the addition of a $(d-1)$-form of the form $n \wedge{ }^{(d-2)} \phi$, where ${ }^{(d-2)} \phi$ is arbitrary. The addition of such a form does however not make any difference in the formulae given below.
} 
where $\zeta^{*}$ denotes the pull-back of a covariant tensor field to $\mathscr{I}$. Thus, it follows from this expression that the pull-back of the symplectic current form to $\mathscr{I}$ is finite and smooth for any linear perturbation preserving our asymptotic flatness condition.

We next look for a potential $\Theta$ for (50). The subsequent calculations are somewhat simplified using the tensor $S_{a b}$ defined by the equation

$$
R_{a b c d}=C_{a b c d}+g_{a[c} S_{d] b}-g_{b[c} S_{d] a}
$$

in terms of the unphysical Riemann tensor and Weyl tensor. It can be expressed in terms of the unphysical Ricci tensor by

$$
S_{a b}=\frac{2}{(d-2)} R_{a b}-\frac{1}{(d-1)(d-2)} R g_{a b}
$$

Using the relation (21) between the physical and unphysical Ricci tensor, we find that Einstein's equation for the physical Ricci tensor takes the form

$$
\Omega S_{a b}+2 \nabla_{a} n_{b}-f g_{a b}=0
$$

in terms of the tensor $S_{a b}$, where we remember the shorthand $f=\Omega^{-1} n^{a} n_{a}=O(1)$. We now take the variation of eq. (53) with respect to a linearized solution of the field equations that preserves asymptotic flatness to first order (remembering $\delta \Omega=0$ since $\Omega$ is rigidly fixed) and substitute the definition of $\tau_{a b}$ and $\tau_{b}$. Using the formulae

$$
\begin{gathered}
\delta f=-\Omega^{\frac{d-2}{2}} \tau_{a} n^{a} \\
\delta\left(\nabla_{a} n_{b}\right)=\Omega^{\frac{d-2}{2}}\left(-\frac{d}{2} n_{(a} \tau_{b)}+\frac{d}{4} f \tau_{a b}+\frac{1}{2} n^{c} \nabla_{c} \tau_{a b}-\Omega \nabla_{(a} \tau_{b)}-\frac{1}{2} \Omega S_{c(a} \tau_{b)}{ }^{c}\right),
\end{gathered}
$$

we find the result

$$
\begin{aligned}
\Omega^{-\frac{d-4}{2}} \delta S_{a b}=d \cdot n_{(a} \tau_{b)}-n^{c} \nabla_{c} \tau_{a b}-\frac{(d-2)}{2} & f \tau_{a b} \\
& +\Omega\left(2 \nabla_{(a} \tau_{b)}+S_{c(a} \tau_{b)}{ }^{c}-\Omega^{-1} \tau_{c} n^{c} g_{a b}\right),
\end{aligned}
$$

and we note that the right side of this equation is manifestly finite at $\mathscr{I}$ and that the term in the second line is of order $\Omega$. It follows from this relation, together with the relation $\tau_{a b} n^{b}=O(\Omega)$, that the pull-back of the symplectic current density at $\mathscr{I}$, eq. (150), can be written as

$$
\zeta^{*} \omega_{a_{1} \ldots a_{d-1}}=\frac{1}{32 \pi G} \Omega^{-\frac{d-4}{2}}\left(\tau_{2}^{c d} \delta_{1} S_{c d}-\tau_{1}^{c d} \delta_{2} S_{c d}\right) \epsilon_{a_{1} \ldots a_{d-1}}
$$


To construct the desired potential, $\Theta$, for eq. (57), we note that our asymptotic flatness conditions (3) and (4) imply that ${ }^{20}$

$$
S_{a b}-\bar{S}_{a b}=O\left(\Omega^{\frac{d-4}{2}}\right), \quad S_{m}^{m}-\bar{S}_{m}^{m}=O\left(\Omega^{\frac{d-2}{2}}\right), \quad f-\bar{f}=O\left(\Omega^{\frac{d}{2}}\right) .
$$

By construction, the restriction of the symplectic form to $\mathscr{I}$, eq. (57), only depends on the physical metric $\tilde{g}_{a b}$ and its variations, but not on how we have chosen to write them in terms of an unphysical metric $g_{a b}$ and a conformal factor $\Omega$, as long as $g_{a b}$ and $\bar{g}_{a b}$ are smooth at $\mathscr{I}$. We now take advantage of this fact by choosing a conformal factor so that the background metric (2) is flat in a neighborhood of $\mathscr{I}$, i.e. $\bar{S}_{a b}=0$, and such that $\bar{f}=$ const. there. This is possible, at least locally in a neighborhood of any open subset of $\mathscr{I}^{+}$resp. $\mathscr{I}^{-}$with compact closure not intersecting spatial infinity (see appendix B for details). Consequently, by eq. (58), in such a gauge we have

$$
S_{a b}=O\left(\Omega^{\frac{d-4}{2}}\right), \quad S_{m}^{m}=O\left(\Omega^{\frac{d-2}{2}}\right), \quad f=\text { const. }+O\left(\Omega^{\frac{d}{2}}\right) .
$$

Choose any smooth covector field $l_{a}$ on $M$ with the property that $l_{a} l^{a}=0, n^{a} l_{a}=+1$ at $\mathscr{I}$, set

$$
q_{a b}=g_{a b}-2 n_{(a} l_{b)}
$$

and define the "news tensor" by

$$
N_{a b}=\zeta^{*}\left(\Omega^{-\frac{d-4}{2}} q^{m}{ }_{a} q_{b}^{n} S_{m n}\right) \quad(\text { assuming } d>4),
$$

where $\zeta^{*}$ denotes the pull back to $\mathscr{I}$. By definition, $N_{a b}$ is a well-defined smooth tensor field at $\mathscr{I}$ with vanishing trace. Using the identity

$$
S_{a b} n^{b}+\nabla_{a} f=0
$$

one sees that $N_{a b}$ is independent of the particular choice of $l^{a}$. A symplectic potential $\Theta$ at $\mathscr{I}$ with the desired properties is now given by

$$
\Theta_{a_{1} \ldots a_{d-1}} \equiv \frac{1}{32 \pi G} \tau^{c d} N_{c d} \epsilon_{a_{1} \ldots a_{d-1}}
$$

While the restriction of the symplectic form $\omega$ to $\mathscr{I}$ (see eq. (57)) only depends on the physical metric and its perturbation, but not on the particular choice of the conformal factor $\Omega$ and unphysical metric (although the latter were used to obtain a convenient form for $\omega$ ), this need not be the case for $\Theta$, as the latter is a potential for $\omega$ and therefore only unique up to a "total variation" $\delta \Pi$. We therefore have to investigate the behavior of $\Theta$ under "conformal gauge changes". If we change the conformal factor to $\Omega^{\prime}=k \Omega$ with some

\footnotetext{
${ }^{20}$ We note that the second relation is in general false in $d=4$.
} 
smooth $k$, then, since the physical metric is to remain unchanged, the unphysical metric changes by $g_{a b}^{\prime}=k^{2} g_{a b}$, and likewise the background metric changes as $\bar{g}_{a b}^{\prime}=k^{2} \bar{g}_{a b}$. The quantities $S_{a b}, n^{a}, f$ change in the following way:

$$
\begin{aligned}
n^{a \prime} & =k^{-1} n^{a}+k^{-2} \Omega \nabla^{a} k, \\
S_{a b}^{\prime} & =S_{a b}+2 k^{-1} \nabla_{a} \nabla_{b} k-k^{-2} g_{a b}\left(\nabla_{m} k\right) \nabla^{m} k, \\
f^{\prime} & =k^{-1} f+2 k^{-2} n^{a} \nabla_{a} k+k^{-3}\left(\nabla^{a} k\right) \nabla_{a} k
\end{aligned}
$$

with similar formulas for the background quantities $\bar{S}_{a b}, \bar{n}^{a}, \bar{f}$. In order to arrive at the above formula for $\Theta$, we assumed that we were in a gauge such that $\bar{S}_{a b}=0$, and such that $\bar{f}=$ const. Since we have thereby already partially fixed the gauge, we need to demand that $k$ be such that $\bar{S}_{a b}^{\prime}=0$ and such that $\bar{f}^{\prime}=$ const. Inserting these formulas, we find that for such $k$

$$
\Theta^{\prime}=\Theta+\delta \Pi,
$$

where $\Pi$ is the $(d-1)$-form on $\mathscr{I}$ defined by

$$
\Pi_{a_{1} \ldots a_{d-1}}=\frac{(d-2)}{2^{6} \pi G} \Omega^{-(d-2)} k^{-1} n^{b}\left(\nabla_{b} k\right)(g-\bar{g})_{c d} q^{c e} q^{d f}(g-\bar{g})_{e f} \epsilon_{a_{1} \ldots a_{d-1}} .
$$

Since $\delta \Pi$ is not vanishing at $\mathscr{I}$, this means that the definition of $\Theta$ is not completely independent upon how the physical metric is written as $\Omega^{2} \tilde{g}_{a b}=g_{a b}$, respectively how the Minkowski metric is written as $\Omega^{2} \tilde{\eta}_{a b}=\bar{g}_{a b}$ in terms of a conformal background metric, with $\bar{S}_{a b}=0$ and $\bar{f}=$ const. We resolve this gauge ambiguity by chosing a representer $\bar{g}_{a b}$ in the conformal class of the Einstein static universe which has

$$
\bar{f}=0, \quad \bar{\nabla}_{a} \bar{n}^{b}=0, \quad \bar{S}_{a b}=0 \quad \text { near } \mathscr{I},
$$

where $\bar{\nabla}_{a}$ denotes the derivative operator associated with $\bar{g}_{a b}$. It can be seen that $\Pi=0$ for any further gauge change preserving this gauge condition, i.e., that $\Theta$ is now defined in a gauge invariant way. We will stick with this gauge choice for the remainder of the paper. It follows from these gauge conditions that

$$
f=O\left(\Omega^{\frac{d}{2}}\right), \quad \nabla_{a} n_{b}=O\left(\Omega^{\frac{d-2}{2}}\right) .
$$

Some further explanations concerning this gauge choice are provided in appendix B.

By the general analysis reviewed in the last section we infer that generators $\mathcal{H}_{\xi}$ associated with asymptotic symmetries $\xi^{a}$ exist in $d$-dimensional general relativity with our choice of asymptotic flatness conditions, and that analysis instructs us to define $\mathcal{H}_{\xi}$ by

$$
\delta \mathcal{H}_{\xi}=\int_{B}\left(\delta Q_{\xi}-\xi \cdot \theta\right)+\frac{1}{32 \pi G} \int_{B} \tau^{a b} N_{a b} \xi \cdot{ }^{(d-1)} \epsilon,
$$

where $Q_{\xi}$ and $\theta$ were defined in eqs. (42) and (34). 
Formulas (70) and (63) are also correct in $d=4$ (for a derivation, see [14]), provided that $N_{a b}$ is given by the usual definition of the news tensor in 4 dimensions, $N_{a b}=$ $\zeta^{*}\left(q^{m}{ }_{a} q_{b}^{n} S_{m n}\right)-\rho_{a b}$, instead of eq. (61). Here, $\rho_{a b}$ is the uniquely determined symmetric tensor on $\mathscr{I}$ provided by thm. 5 of [10], whose precise form depends on the chosen gauge.

Plugging the expression (63) for the symplectic potential into the flux formula, eq. (44), and setting as above $\chi_{a b}=\Omega^{-\frac{d-6}{2}} £_{\xi} \tilde{g}_{a b}$ we get the following expression for the flux associated with an asymptotic symmetry $\xi^{a}$ through a segment $\mathscr{S}$ of $\mathscr{I}$

$$
F_{\xi}=\frac{1}{32 \pi G} \int_{\mathscr{S}} \chi^{c d} N_{c d}{ }^{(d-1)} \epsilon
$$

noting that this is finite on account of our definition of an asymptotic symmetry, see eqs. (10).

Having established the existence of a generator $\mathcal{H}_{\xi}$, we now discuss its uniqueness. The definition of $\mathcal{H}_{\xi}$ depends on the choice of $\Theta$, which is itself only unique up to the addition of a $(d-1)$-form on $\mathscr{I}$ of the form $\delta W$, where $W$ is an arbitrary $(d-1)$-form on $\mathscr{I}$ that is locally constructed out of the physical metric, the physical Riemann tensor and its derivatives, and $\Omega$. The change $\Omega \rightarrow \lambda \Omega$ and $g_{a b} \rightarrow \lambda^{2} g_{a b}$ with $\lambda$ a constant will keep the physical metric fixed and preserve (69), so gauge invariance requires that $W \rightarrow W$ under this change of the unphysical metric and the conformal factor. (This requirement is met by our definition (63) of $\Theta$.) Moreover, the symplectic potential $\Theta$ defined in (63) has the property that it vanishes whenever the news tensor, $N_{a b}$, vanishes. A vanishing news tensor indicates the absence of radiation (at least in 4 dimensions), and our definition (63) for $\Theta$ has the property that it vanishes when $N_{a b}=0$, thereby implying by eq. (44) that the flux also vanishes whenever the news vanishes. It is natural to demand that any reasonable definition of $\Theta$, and hence the flux, vanishes when the news is zero, which in turn leads to the requirement that $W=0$ whenever $N_{a b}=0$. If $W$ has furthermore an analytic dependence on the (physical) metric, then we claim that these requirements imply that $W=0$, and hence that $\Theta$ is unique.

In order to see that this is indeed true, it is useful to introduce the "scaling dimension" [10] of a tensor $L^{a \ldots b}{ }_{c \ldots d}$ with $u$ upper indices and $l$ lower indices that is constructed out of the unphysical metric and $\Omega$. We say that such a tensor has scaling dimension $s$ if $L^{a \ldots b}{ }_{c \ldots d} \rightarrow \lambda^{s-u+l} L^{a \ldots b}{ }_{c \ldots d}$ under a change $\Omega \rightarrow \lambda \Omega$ and $g_{a b} \rightarrow \lambda^{2} g_{a b}$. It follows from this definition that the scaling dimension does not depend on the position of the indices and is additive under the tensor product. The dimension of $g_{a b}$ is 0 , the dimension of $\Omega$ is +1 , the dimension of the Riemann tensor is -2 and each derivative decreases the dimension by 1 , which implies that the dimension of $n_{a}$ is 0 . By assumption, the $(d-1)$ form

$$
W_{a b \ldots c}=Y\left(\Omega, g_{a b}, n_{a}, \ldots,\left(\nabla_{m}\right)^{r} n_{a}, R_{a b c d}, \ldots,\left(\nabla_{m}\right)^{t} R_{a b c d}\right)^{(d-1)} \epsilon_{a b \ldots c}
$$

has scaling dimension $-(d-1)$. Therefore, since ${ }^{(d-1)} \epsilon$ has scaling dimension $0, Y$ must have scaling dimension $-(d-1)$. Using Einstein's equation to eliminate covariant derivatives of $n_{a}$ in terms of covariant derivatives or $S_{a b}$, and using eq. (51) to eliminate the 
Riemann tensor in favor of $C_{a b c d}$ and $S_{a b}$, we can write a term in $Y$ schematically in the form

$$
\Omega^{v}\left(n_{a}\right)^{l} \prod_{i=1}^{r}\left(\nabla_{m}\right)^{s_{i}}\left(\Omega^{-\frac{d-4}{2}} S_{a b}\right) \prod_{j=1}^{u}\left(\nabla_{m}\right)^{t_{j}}\left(\Omega^{-\frac{d-6}{2}} C_{a b c d}\right),
$$

where we have suppressed contractions with the metric $g_{a b}$ to lighten the notation. The scaling dimension of this term must be equal $-(d-1)$, which implies that

$$
\sum_{i} s_{i}+\frac{d}{2} r+\sum_{j} t_{j}+\frac{(d-2)}{2} u-v=d-1 .
$$

Since the expressions $\Omega^{-\frac{d-4}{2}} S_{a b}$ and $\Omega^{-\frac{d-6}{2}} C_{a b c d}$ are smooth at $\mathscr{I}$ as a consequence of our definition of asymptotic flatness, the expression (73) can be nonvanishing at $\mathscr{I}$ if and only if $v \leq 0$ (note that $v<0$ is allowed, since the other terms appearing in the above expression could vanish at $\mathscr{I})$. Furthermore, $\Omega^{-\frac{d-4}{2}} S_{a b}$ vanishes at $\mathscr{I}$ if and only if the news vanishes at $\mathscr{I}$. Therefore, since we want $Y$ to vanish whenever $N_{a b}=0$, we must have $r>0$. On the other hand, eq. (74) implies that $r \leq 1$, so $r=1$. We now analyze the remaining cases: When $r=1$ and $u=0$, then the term (73) looks schematically like

$$
\Omega^{v}\left(n_{a}\right)^{l}\left(\nabla_{m}\right)^{s}\left(\Omega^{-\frac{d-4}{2}} S_{a b}\right),
$$

with $s-v=(d-2) / 2$. This term has to vanish when the news vanishes and hence when $\Omega^{-\frac{d-4}{2}} S_{a b}$, but not necessarily its derivatives, vanishes at $\mathscr{I}$. This implies $s=0$ and hence $v=-(d-2) / 2$, so we need contractions of $n_{a}$ with itself to get a term that is finite at $\mathscr{I}$. But contractions of $n_{a}$ with itself give a power of at least $\Omega^{\frac{d+2}{2}}$, therefore terms of the form eq. (75) cannot occur. The only remaining nontrivial case is $r=1$ and $u=1$. In this case, we must have $s_{i}=t_{j}=v=0$ and the term (73) must take the form $\Omega^{-(d-5)} C_{a b c d} S^{a c} n^{b} n^{d}$. But this term vanishes at $\mathscr{I}$, by eq. (115) in the appendix. We have therefore shown that $W=0$ and hence that the symplectic potential $\Theta$ given by eq. (63) is unique under the above assumptions.

We now consider the flux for the special case of "translational" asymptotic symmetries $\xi^{a}$. These are distinguished by the fact that the restriction of $\xi^{a}$ to $\mathscr{I}$ is proportional to $n^{a}$, i.e., $\xi^{a}=\alpha n^{a}+\Omega k^{a}$, for some $k^{a}$, smooth at $\mathscr{I}$. A vector field $\xi^{a}$ is an asymptotic symmetry if and only if the tensor $\chi_{a b}=\Omega^{-\frac{d-6}{2}} £_{\xi} \tilde{g}_{a b}$ satisfies eqs. (10). If we substitute this form of $\xi^{a}$ into eqs. (10), we see that $k^{a}=-\nabla^{a} \alpha$ at $\mathscr{I}$. Let us therefore make the ansatz

$$
\xi^{a}=\alpha n^{a}-\Omega \nabla^{a} \alpha
$$

For which $\alpha$ is this an asymptotic symmetry? Inserting (76) into $\chi_{a b}$, we find that

$$
\chi_{a b}=2 \Omega^{-\frac{d-4}{2}}\left(-\nabla_{a} \nabla_{b} \alpha+\Omega^{-1} \alpha \nabla_{a} n_{b}+\Omega^{-1} g_{a b} n^{c} \nabla_{c} \alpha\right) .
$$


Using that $\nabla_{a} n_{b}=O\left(\Omega^{\frac{d-2}{2}}\right)$ in our gauge choice (see eq. (69) $)$ we see that $\chi_{a b}$ is finite at $\mathscr{I}$ if and only if $\alpha$ satisfies

$$
\nabla_{a} \nabla_{b} \alpha-\Omega^{-1} g_{a b} n^{c} \nabla_{c} \alpha=O\left(\Omega^{\frac{d-4}{2}}\right)
$$

Dotting $n^{a}$ into eq. (77), and using that $f=O\left(\Omega^{\frac{d}{2}}\right)$ in our gauge choice, we see that $\chi_{a b} n^{b}=O(\Omega)$ if and only if

$$
\nabla^{b}\left(\Omega^{-1} n^{a} \nabla_{a} \alpha\right)=O\left(\Omega^{\frac{d-4}{2}}\right)
$$

Contracting this once more into $n^{a}$, we see that $\chi_{a b} n^{a} n^{b}=O\left(\Omega^{2}\right)$ if

$$
\Omega^{-1} n^{b} \nabla_{b}\left(\Omega^{-1} n^{a} \nabla_{a} \alpha\right)=O\left(\Omega^{\frac{d-4}{2}}\right)
$$

In $d>4$, an asymptotic symmetry must furthermore satisfy $\chi_{a}^{a}=O(\Omega)$. However, this condition actually automatically follows for any asymptotic symmetry $\xi^{a}$ once $\chi_{a b}=$ $O(1), \chi_{a b} n^{b}=O(\Omega), \chi_{a b} n^{a} n^{b}=O\left(\Omega^{2}\right)$ are satisfied. To see this, we note that since $\delta \tilde{g}_{a b}=£_{\xi} \tilde{g}_{a b}$ satisfies the linearized Einstein equation, the tensor $\chi_{a b}$ satisfies eq. (22) (with $\tau_{a b}=\chi_{a b}$ in that equation). Multiplying eq. (22) by $\Omega$, we see that the only remaining singular term on the right side is given by a constant that is nonzero for $d \neq 4$, times $\Omega^{-1} n_{a} n_{b} \chi_{c}{ }^{c}$, which implies that $\chi_{a}{ }^{a}=O(\Omega)$ when $d>4$. Thus, if eqs. (78), (79) and (80) hold, then the vector field $\xi^{a}=\alpha n^{a}-\Omega \nabla^{a} \alpha$ is an asymptotic symmetry.

The above conditions on $\alpha$ can be understood as follows. In $d=4$ dimensions, conditions (78) and (79) together imply that $\alpha$ must be constant along the null generators of $\mathscr{I}$, whereas condition (80) gives a restriction on how $\alpha$ is defined off of $\mathscr{I}$. Hence, $\alpha$ is essentially an arbitrary function on a given cross section of $\mathscr{I}$, which is propagated along the null generator to the other cross sections. The corresponding symmetries are commonly referred to as "supertranslations". They comprise the ordinary "pure" translations, as well as an additional infinite set of mutually commuting so-called "angle dependent" translations. In $d>4$, the above conditions are more restrictive than in $d=4$ and are analyzed in appendix C. There are now only $d$ linearly independent admissible functions $\alpha$ up to correction terms which essentially do not affect the restriction of $\chi_{a b}$ to $\mathscr{I}$, in the sense that the correction terms do not make a contribution to the flux. The translational asymptotic symmetries associated with these choices of $\alpha$ correspond to the $d$ translational Killing fields in Minkowski spacetime. There is no direct analog of the angle dependent translations in higher dimensions. The asymptotic translations with $\alpha \geq 0$

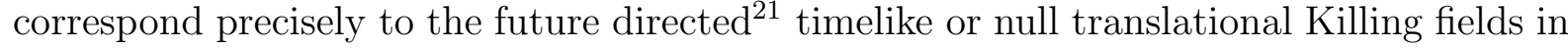
Minkowski spacetime.

Let us calculate the flux when $\xi^{a}=\alpha n^{a}-\Omega \nabla^{a} \alpha$ is an asymptotic future directed time translation, i.e. $\alpha \geq 0$. Using Einstein's equation (53) to eliminate the term proportional

\footnotetext{
${ }^{21} \alpha \geq 0$ means that $\xi^{a}=\alpha n^{a}$ is future pointing near $\mathscr{I}^{+}$.
} 
to $\nabla_{a} n_{b}$ in eq. (77) in favor of $S_{a b}$, we can bring $\chi_{a b}$ into the form

$$
\chi_{a b}=-\Omega^{-\frac{d-4}{2}}\left(2 \nabla_{a} \nabla_{b} \alpha+\alpha S_{a b}-2 \Omega^{-1} g_{a b} n^{c} \nabla_{c} \alpha-\alpha \Omega^{-1} f g_{a b}\right) .
$$

Substituting it into the flux formula, eq. (71), one finds

$$
F_{\xi}=-\frac{1}{32 \pi G} \int_{\mathscr{S}} \alpha N^{c d} N_{c d}{ }^{(d-1)} \epsilon \leq 0 .
$$

This shows that the flux of energy (defined via any future directed asymptotic time translation) through $\mathscr{I}$ is always negative, i.e., that the energy radiated away by the system is always positive.

The generators $\mathcal{H}_{\xi}$ are determined, in principle, by the defining relation eq. (70) and the requirement that $\mathcal{H}_{\xi}=0$ on Minkowski spacetime. If $\xi^{a}$ is not a translation, i.e., if $\xi^{a}$ is tangent to some cross section $B$ of $\mathscr{I}$, then the term involving $\Theta$ vanishes in the expression for the variation of $\mathcal{H}_{\xi}$, eq. (70), and an explicit expression for $\mathcal{H}_{\xi}$ can be derived in basically the same manner as in 4 dimensions, see [14. We will not discuss this case here but focus on the case when $\xi^{a}$ is a translation for the rest of this section.

In that case, the defining relation (170) is not useful to actually find the expression for the generators $\mathcal{H}_{\xi}$, although the right side of that equation is, of course, explicitly known. Indeed, in [14, an explicit expression for $\mathcal{H}_{\xi}$ in $d=4$ was found by verifying that relation (170) is satisfied by a known expression for the Bondi energy previously given by Geroch [10]. Such a candidate expression is, of course, not available in $d>4$, since this is precisely what we are actually looking for in the first place. We therefore proceed by a different route, restricting ourselves for simplicity first to the case of the asymptotic translation $\xi^{a}=\alpha n^{a}$, with $\alpha$ a constant.

Consider the $(d-1)$-form $\Theta\left(\tilde{g} ; £_{\alpha n} \tilde{g}\right)$ on $\mathscr{I}$ that is given by the integrand of the flux integral, eq. (82). We extend this to a $(d-1)$-form that is defined on the entire unphysical spacetime $M$ by setting

$$
\Theta_{s_{1} \ldots s_{d-1}}=\frac{1}{32 \pi G} \alpha \Omega^{-(d-4)}\left(S_{a b} S_{c d} q^{a c} q^{b d}\right) \epsilon_{s_{1} \ldots s_{d-1}} .
$$

Define the vector field

$$
P^{a} \equiv \frac{\alpha}{8(d-3) \pi G} \Omega^{-(d-4)}\left(S_{d e} q^{c e} q^{d[b} n^{a]} \nabla_{b} l_{c}-\Omega^{-1} C^{a b c d} n_{b} l_{c} n_{d}\right)
$$

in the interior of the spacetime, where $l_{a}$ is any smooth vector field such that $l_{a} l^{a}=0$ and $n^{a} l_{a}=1$ on $\mathscr{I}$ and such that relations (103) are satisfied. We show in the appendix that

$$
\nabla^{a} P_{a}=\frac{1}{32 \pi G} \alpha \Omega^{-(d-4)} S_{a b} S_{c d} q^{a c} q^{b d}+O(\Omega)
$$


and it can be verified directly from the definition of $P^{a}$ that $P^{a} n_{a}=O\left(\Omega^{2}\right)$. Next, define the $(d-2)$-form $\mu$ by $^{22}$

$$
\mu_{a_{1} \ldots a_{d-2}}=\epsilon_{a_{1} \ldots a_{d-2} c d} l^{c} P^{d}=\frac{1}{2}[*(l \wedge P)]_{a_{1} \ldots a_{d-2}} .
$$

Then it follows that

$$
\begin{aligned}
(\mathrm{d} \mu)_{a_{1} \ldots a_{d-1}} & =2 \nabla_{m}\left(P^{[m} l^{n]}\right) \epsilon_{n a_{1} \ldots a_{d-1}} \\
& =2 n_{n} \nabla_{m}\left(P^{[m} l^{n]}\right) \epsilon_{a_{1} \ldots a_{d-1}}-2(d-1) \nabla_{m}\left(P^{[m} l^{n]}\right) n_{\left[a_{1}\right.} \epsilon_{\left.|n| a_{2} \ldots a_{d-1}\right]} \\
& =\left(\nabla_{m} P^{m}\right) \epsilon_{a_{1} \ldots a_{d-1}}-2(d-1) \nabla_{m}\left(P^{[m} l^{n]}\right) n_{\left[a_{1}\right.} \epsilon_{\left.|n| a_{2} \ldots a_{d-1}\right]}+O(\Omega), \\
& =(\Theta+\mathrm{d} \Omega \wedge \varphi)_{a_{1} \ldots a_{d-1}}+O(\Omega),
\end{aligned}
$$

where $\epsilon_{a_{1} \ldots a_{d-1}}$ is as in eq. (49), where it has been used that $P^{a} n_{a}=O\left(\Omega^{2}\right)$, and where we have set $\varphi_{a_{1} \ldots a_{d-2}}=2 \nabla_{m}\left(P^{[m} l^{n]}\right) \epsilon_{n a_{1} \ldots a_{d-2}}$.

Consider now a segment $\mathscr{S}$ of $\mathscr{I}$ bounded by cross sections $B_{1}$ and $B_{2}$, and a sequence of smooth $(d-1)$-surfaces $\mathscr{S}_{i}$ of constant $\Omega$ that approach $\mathscr{S}$. Using eqs. (44) and (87), we can write the flux through the segment $\mathscr{S}$ as follows:

$$
\begin{aligned}
F_{\alpha n}=\lim _{i \rightarrow \infty} \int_{\mathscr{S}_{i}} \Theta\left(\tilde{g}, £_{\alpha n} \tilde{g}\right) & =\lim _{i \rightarrow \infty} \int_{\mathscr{S}_{i}}(\mathrm{~d} \mu-\mathrm{d} \Omega \wedge \varphi) \\
& =\lim _{i \rightarrow \infty}\left(\int_{\left(\partial \mathscr{S}_{i}\right)_{2}} \mu-\int_{\left(\partial \mathscr{S}_{i}\right)_{1}} \mu\right)
\end{aligned}
$$

where we have used Stoke's theorem ${ }^{23}$ and where we have written $\left(\partial \mathscr{S}_{i}\right)_{1}$ for the connected component of the boundary approaching $B_{1}$ and $\left(\partial \mathscr{S}_{i}\right)_{2}$ for the connected component of the boundary approaching $B_{2}$. Now take the variation of this equation and substitute the variation of the flux formula (43) $), \delta F_{\alpha n}=\delta \mathcal{H}_{\alpha n}\left(B_{1}\right)-\delta \mathcal{H}_{\alpha n}\left(B_{2}\right)$. This gives

$$
\delta \mathcal{H}_{\alpha n}\left(B_{1}\right)-\delta \mathcal{H}_{\alpha n}\left(B_{2}\right)=\lim _{i \rightarrow \infty}\left(\delta \int_{\left(\partial \mathscr{S}_{i}\right)_{2}} \mu-\delta \int_{\left(\partial \mathscr{S}_{i}\right)_{1}} \mu\right) .
$$

Consider a variation of the metric that vanishes in a neighborhood of some cross section of $\mathscr{I}$, in addition to satisfying the linearized equations of motion and the linearized conditions of asymptotic flatness. Then it follows from eq. (89) that for such a variation,

$$
\delta \mathcal{H}_{\alpha n}(B)=\delta \int_{B} \mu
$$

\footnotetext{
${ }^{22}$ Our convention for the $*$ operation of a $p$-form is $(* A)_{a_{1} \cdots a_{d-p}}=\epsilon_{a_{1} \cdots a_{d-p}} b_{1} \cdots b_{p} A_{b_{1} \cdots b_{p}}$.

${ }^{23} \mathrm{~A}$ subtlety arises from the fact that the gauge that we are working in (chosen such that eq. (69) holds) is actually not defined on all of $\mathscr{I}$, but only on $\mathscr{I}$ minus a single generator, see appendix B. Therefore, there also ought to appear another "boundary term" in eq. 888 corresponding to that single generator. However, it can be seen that this term does not make a contribution by passing to a suitable gauge which is defined on all of $\mathscr{I}$, and by transforming the expression for $\mu$ accordingly using formulas very similar to those given on p. 50-51 of [9]. An example is worked out in appendix B. Similar remarks apply to other formulas below.
} 
for any cross section $B$ of $\mathscr{I}$, where the integral on the right side is defined by the limit of the corresponding integrals over $(d-2)$-surfaces of constant $\Omega$ that smoothly approach $B$ from the interior of the spacetime ${ }^{24}$. Next, consider a variation that is pure gauge, $\delta \tilde{g}_{a b}=£_{\eta} \tilde{g}_{a b}$, for some asymptotic symmetry $\eta^{a}$. For such a variation, we have

$$
\begin{aligned}
\delta \int_{B} \mu & =\int_{B} £_{\eta} \mu \\
& =\int_{B}[\mathrm{~d}(\eta \cdot \mu)+\eta \cdot \mathrm{d} \mu] \\
& =\int_{B} \eta \cdot \Theta\left(\tilde{g} ; £_{\alpha n} \tilde{g}\right),
\end{aligned}
$$

where the integrals are defined by a limiting procedure as above. We now show that $\delta \mathcal{H}_{\alpha n}(B)$ for this variation is also given by the right side of the above equation. For this, we consider the 1-parameter family of diffeomorphisms $\Phi_{t}$ generated by $\eta^{a}$ which maps points in $\mathscr{I}$ to points in $\mathscr{I}$. If $\Phi_{t}^{*} B$ is the cross section of $\mathscr{I}$ obtained from $B$ by applying this diffeomorphism, and if $\mathscr{S}_{t}$ is the segment of $\mathscr{I}$ bounded by these two cross sections, then we have

$$
\begin{aligned}
\delta \mathcal{H}_{\alpha n}(B) & =\lim _{t \rightarrow 0} \frac{1}{t}\left[\mathcal{H}_{\alpha n}(B)-\mathcal{H}_{\alpha n}\left(\Phi_{t}^{*} B\right)\right] \\
& =\lim _{t \rightarrow 0} \frac{1}{t} \int_{\mathscr{S}_{t}} \Theta\left(\tilde{g} ; £_{\alpha n} \tilde{g}\right) \\
& =\int_{B} \eta \cdot \Theta\left(\tilde{g} ; £_{\alpha n} \tilde{g}\right),
\end{aligned}
$$

for the variation $\delta \tilde{g}_{a b}=£_{\eta} \tilde{g}_{a b}$, where we have used the flux formula eq. (44) in the second line, and where we have used that $\Theta\left(\tilde{g} ; £_{\alpha n} \tilde{g}\right)$ is smooth at $\mathscr{I}$ in the third line. Hence, we conclude that eq. (90) also holds for any variation of the form $\delta \tilde{g}_{a b}=£_{\eta} \tilde{g}_{a b}$. Consider now a variation with compact support $K$ on some Cauchy surface which satisfies the linearized field equations and the linearized conditions of asymptotic flatness. Then it follows from the arguments given in section 3 that such a variation can be written as a sum $\delta \tilde{g}_{a b}+£_{\eta} \tilde{g}_{a b}$, where $\eta^{a}$ is some asymptotic symmetry and where $\delta \tilde{g}_{a b}$ has support in $J^{+}(K) \cup J^{-}(K)$. It follows that there exist cross sections $B^{+}$of $\mathscr{I}^{+}$and $B^{-}$of $\mathscr{I}^{-}$such that $\delta \tilde{g}_{a b}$ vanishes in a neighborhood of $B^{+}$and $B^{-}$. By the arguments already given, eq. (90) therefore holds for any variation which has compact support on some Cauchy surface. Consequently, eq. (90) will also hold for any variation such that the corresponding fields $\tau_{a b}, \tau_{a}$ and their first (unphysical) derivatives can be approximated near $B$ by the corresponding fields for

\footnotetext{
${ }^{24}$ This shows in particular that the right side of eq. (90) (defined via this limiting procedure) is actually finite. This is not obvious from the definition since $P^{a}$ as well as its variation is not manifestly finite at $\mathscr{I}$ in $d>4$. Indeed, the Weyl term in the definition of $P^{a}$ can only be shown to make a contribution of order $O\left(\Omega^{-(d-4) / 2}\right)$ using our asymptotic flatness conditions.
} 
a sequence of variations that have compact support on a Cauchy surface. We believe that all variations that satisfy the linearized equations of motion and the linearized conditions of asymptotic flatness can be approximated in this way. Assuming that this is true, it follows that eq. (90) holds for all variations.

We have $\int_{B} \bar{\mu}=0$ for our background geometry, since we are in a gauge in which $\bar{g}_{a b}$ is flat in a neighborhood of $\mathscr{I}$, therefore $\bar{\mu}=0$ in a neighborhood of $\mathscr{I}$. Therefore, modulo the proof of the approximation property mentioned in the last paragraph, we have shown that

$$
\mathcal{H}_{\xi}=\int_{B} \mu=\frac{1}{2} \int_{B} *(l \wedge P)
$$

for asymptotic time translations, $\xi^{a}=\alpha n^{a}$, where $P^{a}$ given by eq. (84). This is our expression for the Bondi energy of an asymptotically flat spacetime in $d$ dimensions.

Our expression for the Bondi energy is independent of the particular choice of $l_{a}$ with the properties (103): Consider another $l_{a}^{\prime}$ with the same properties as $l_{a}$, and set $x_{a}=l_{a}-l_{a}^{\prime}$. Then $x_{a}$ satisfies the relations $\nabla_{b} x_{a}=O\left(\Omega^{\frac{d-4}{2}}\right), n_{a} x^{a}=O\left(\Omega^{\frac{d}{2}}\right)$ and $l^{a} x_{a}=O\left(\Omega^{\frac{d-2}{2}}\right)$. Consider the antisymmetric tensor field $X^{a b}$ defined by

$$
X^{a b}=\frac{1}{8(d-3) \pi G} \alpha \Omega^{-(d-4)} S_{e f} q^{e d} q^{f[a} n^{b]} x_{d}
$$

Then it can be seen, using formulas (103), (69) and (107), that $X^{a b} n_{b}=O(\Omega)$,

$$
P^{\prime a}=P^{a}+\nabla_{b} X^{a b}+O(\Omega) .
$$

Consider now a sequence of embedded $(d-2)$-surfaces that are smoothly embedded into $M$ and which approach a cross section $B$ of $\mathscr{I}$ as $i \rightarrow \infty$. Without loss of generality we may assume that $\Omega=$ const. on each of these surfaces, so that $n^{a}$ is one of the normals to the surfaces. Let $u^{a}$ be another normal so that the field $n^{[a} u^{b]}$ is a binormal, meaning that $\epsilon_{a_{1} \ldots a_{d-2}}=\zeta_{i}^{*}\left(n^{[m} u^{n]} \epsilon_{m n a_{1} \ldots a_{d-2}}\right)$ is equal to the $(d-2)$-volume form induced by $g_{a b}$ on each of these surfaces, where $\zeta_{i}^{*}$ denotes the pull back to $B_{i}$. Then from eq. (95), we get

$$
\begin{aligned}
\zeta_{i}^{*}\left(\mu-\mu^{\prime}\right)_{a_{1} \ldots a_{d-2}}= & \frac{3}{2} \zeta_{i}^{*} \nabla_{q}\left(X^{[q m} l^{n]}\right) \epsilon_{m n a_{2} \ldots a_{d-2}} \\
& -\zeta_{i}^{*}\left(\frac{3}{2} X^{[m q} \nabla_{q} l^{n]}+\frac{1}{2} l^{q} \nabla_{q} X^{m n}+P^{\prime[m} x^{n]}\right) n_{m} u_{n} \epsilon_{a_{1} \ldots a_{d-2}} .
\end{aligned}
$$

But the terms in the last line are all of $O(\Omega)$, since $X^{a b}=O\left(\Omega^{\frac{d-4}{2}}\right), X^{a b} n_{b}=O\left(\Omega^{2}\right)$, $P^{a} n_{a}=O\left(\Omega^{2}\right)$, and since $x^{a} n_{a}=O\left(\Omega^{\frac{d}{2}}\right), n^{b} \nabla_{a} l_{b}=O\left(\Omega^{\frac{d-2}{2}}\right)$ by eqs. (103). This shows that, in differential forms notation,

$$
\zeta_{i}^{*}\left(\mu-\mu^{\prime}\right)=-\frac{1}{3} \zeta_{i}^{*}\{\mathrm{~d}[*(X \wedge l)]\}+O(\Omega)
$$


and therefore, by Stoke's theorem, that

$$
\int_{B} \mu-\int_{B} \mu^{\prime}=\lim _{i \rightarrow \infty} \int_{B_{i}}\left(-\frac{1}{3} \mathrm{~d}[*(X \wedge l)]+O(\Omega)\right)=0
$$

since $B_{i}$ has no boundary. Thus, our definition (93) of the Bondi energy does not depend on our choice of $l_{a}$.

Substituting our expression (84) for $P^{a}$ into eq. (93), using the definition of $S_{a b}$ together with the fact that $S_{m}{ }^{m}=O\left(\Omega^{\frac{d-2}{2}}\right)$, we can express $\mathcal{H}_{\xi}$ by the final formula

$$
\begin{array}{r}
\mathcal{H}_{\xi}=\frac{1}{8(d-3) \pi G} \int_{B} \alpha \Omega^{-(d-4)}\left(\frac{1}{(d-2)} R_{a b} q^{a c} q^{b d}\left(\nabla_{c} l_{d}\right) n^{e} l^{f}\right. \\
\left.-\Omega^{-1} l^{[e} C^{f] b c d} n_{b} l_{c} n_{d}\right) \epsilon_{e f a_{1} \ldots a_{d-2}}
\end{array}
$$

This formula holds for the special translation $\xi^{a}=\alpha n^{a}$, with $\alpha=$ const. The above arguments and calculations can be generalized to arbitrary (null) translations, $\xi^{a}=\alpha n^{a}-$ $\Omega \nabla^{a} \alpha$. One now finds the formula

$$
\begin{aligned}
\mathcal{H}_{\xi}=\frac{1}{8(d-3) \pi G} & \int_{B} \Omega^{-(d-4)}\left(\frac{1}{(d-2)} R_{a b} q^{a c} q^{b d}\left(\nabla_{c} l_{d}\right) \xi^{e} l^{f}\right. \\
& \left.-\Omega^{-1} \alpha^{-1}\left(l^{[e}-v \alpha^{-1} \nabla^{[e} \alpha\right) C^{f] b c d} \xi_{b}\left(l_{c}-v \alpha^{-1} \nabla_{c} \alpha\right) \xi_{d}\right) \epsilon_{e f a_{1} \ldots a_{d-2}}
\end{aligned}
$$

where $v$ is a function such that $\nabla_{a} v=l_{a}$. It can be verified again that this expression does not depend on the particular choice of $l_{a}$. Formula (100) can alternatively be derived by noting that any null translation $\xi^{a}$ can be obtained from $n^{a}$ by applying an asymptotic symmetry, $\xi^{a}=\phi^{*} n^{a}$. Since the Bondi energy for the vector field $\xi^{a}$ and metric $\tilde{g}_{a b}$ evaluated at $B$ is equal to the Bondi energy for $n^{a}=\xi^{a \prime}=\phi^{-1 *} \xi^{a}$ and metric $\tilde{g}_{a b}^{\prime}=$ $\phi^{-1 *} \tilde{g}_{a b}$ evaluated at the cross section $B^{\prime}=\phi(B)$, one can obtain the Bondi energy for $\xi^{a}$ by applying eq. (99) to the metric $\tilde{g}_{a b}^{\prime}$ and the cross section $B^{\prime}$. The above expression (100) is then obtained using the formulae $\phi^{*} g^{\prime}{ }_{a b}=\alpha^{-2} g_{a b}, \phi^{*} \Omega=\alpha^{-1} \Omega, \phi^{*} l^{a \prime}=\alpha l^{a}-v \nabla^{a} \alpha$, $\phi^{*} n^{a \prime}=\alpha n^{a}-\Omega \nabla^{a} \alpha$, as well as our asymptotic flatness conditions.

Equation (100) is the main result of our paper. It holds in the gauge defined in (68). The corresponding formula for other choices of the background geometry $\bar{g}_{a b}$ can be obtained by applying the corresponding gauge transformation to our formula. In the case $d=4$, formula (100) is not correct. An expression in $d=4$ has been given by Geroch [10]. It involves, among other things, the news tensor (given by $N_{a b}=S_{a b}-\rho_{a b}$ in $d=4$ ), instead of the unphysical Ricci tensor. 
The first and second term in the integrand of (100) can be roughly interpreted as follows: the second term is the "Coulomb part" of the Weyl tensor (multiplied by suitable powers of $\Omega$ ), and represents the "pure Coulomb contribution" to the Bondi energy. The first term represents contributions from gravitational radiation; it follows from eq. (103) given in appendix $\mathrm{A}$ that it vanishes if and only if the news tensor, $N_{a b}$, and hence the flux, vanishes. In 4 dimensions, it can be proven [10] that the news tensor, and hence the radiative contribution to the Bondi energy, always vanishes in stationary spacetimes. It would be interesting to see whether an analog of this result holds in $d$ dimensions.

In the $d$-dimensional analog of Schwarzschild spacetime given by the line element (15), the Bondi energy is evaluated as follows. The term involving $R_{a b}$ in our expression (100) for the Bondi energy does not contribute, showing that there is no radiative contribution to the Bondi energy. The Coulomb contribution is found to be $\Omega^{-(d-3)} C^{a b c d} l_{a} n_{b} l_{c} n_{d}=$ $c(d-2)(d-3) / 4$ at $\mathscr{I}$. Normalizing $\alpha$ so that $\alpha n^{a}-\Omega \nabla^{a} \alpha$ is equal to the timelike Killing field $t^{a}$ of the metric (5) at infinity gives

$$
\mathcal{H}_{\alpha n}=\frac{c(d-2) A_{d-2}}{16 \pi G} \quad\left(=\frac{c}{2 G} \text { in } 4 \text { dimensions }\right)
$$

where $A_{d-2}$ is the area of the unit sphere $S^{d-2}$. This coincides with the ADM mass of the spacetime (5) (given e.g. in [20]), as we expect.

\section{Conclusions}

We have given a geometrical definition of the asymptotic flatness at null infinity in spacetimes of even dimension $d$ greater than 4 within the framework of conformal infinity. Our definition was shown to be stable against perturbations to linear order and was shown to be stringent enough to allow one to define the total energy of the system viewed from null infinity as the generator conjugate to an asymptotic time translation. We proposed to take this notion of energy as the natural generalization of the Bondi energy to higher dimensions. Our definitions of asymptotic flatness and the Bondi energy differ qualitatively from the corresponding definitions in $d=4$; although the asymptotic structure of null infinity in higher dimensions parallels that in 4-dimensions in some ways, the latter seems to be a rather special case on the whole compared to general $d>4$.

Our definitions and constructions related to asymptotic flatness and Bondi energy do not work in odd spacetime dimensions, essentially because the unphysical metric seems to have insufficient regularity properties at null infinity in that case. The case of odd dimensional theories of gravity therefore remains open. Apart from this issue, the analysis given in this paper could be generalized in two obvious ways: (1) by including matter fields, and (2) by admitting higher derivative terms such as the square of the scalar curvature in the gravitational action. 
With regard to the first possibility, one would first have to formulate appropriate asymptotic conditions on the matter fields, which in practice would presumably be found by performing a perturbation analysis. We expect the analysis given in section 3 of this paper to generalize straightforwardly to include conformally invariant fields such as a conformally coupled scalar field, or a an abelian $p$-form gauge field $A$ [with Lagrangian density $L=\mathrm{d} A \wedge *(\mathrm{~d} A)]$ in $d=2 p+2$ spacetime dimensions. This kind of analysis should also still work for other (non-conformally invariant) massless fields. For massive fields a different kind of analysis is probably needed, although we expect on physical grounds that these fields have the best (i.e., exponential) drop off behavior at null infinity. Altogether, we expect that the asymptotic conditions for the combined metric and matter fields are given by the conditions given in section 2 for the metric, plus a condition of the form $T_{a b}=O\left(\Omega^{s}\right)$ for the stress energy of the matter fields, where $s$ is a suitable number. With these conditions in place, a derivation of the Bondi energy can presumably be given in close parallel to our analysis in section 5.

With regard to the second possibility, it is much less clear to us what the likely asymptotic conditions on the gravitational fields might be in that case, or even how they depend on the actual form of the Lagrangian. In fact, it is not even clear to us that there will generically be any reasonable definition of asymptotic flatness that is stable under linear perturbations. Moreover, the linearized equations will now have more derivatives and are therefore presumably harder to analyze than the linearized Einstein's equations. One may ignore the issue of stability and simply try to repeat the analysis of this paper and [14] using the asymptotic flatness conditions of section 2 which have been shown to work for general relativity. However, even though an expression for the Bondi energy might be found in this way, its physical significance would be far from clear under these circumstances.

Acknowledgements: We would like to thank Bob Wald for discussions, especially with regard to the definition of asymptotic flatness in higher dimensions. We also thank Gary Gibbons for conversations. We benefited from talks and conversations during the YITP workshop, YITP-W-02-19 on "Extra dimensions and Braneworld" held at the Yukawa Institute for Theoretical Physics at Kyoto University. A.I. thanks the Enrico Fermi Institute for hospitality. This work was supported in part by NFS grant PHY0090138 to the University of Chicago (S.H.) and by the Japan Society for the Promotion of Science (A.I.). 


\section{A Derivation of equation (84)}

In this appendix we derive expression (84) for $P^{a}$ as a solution to the equation

$$
\nabla^{a} P_{a}=\frac{1}{32 \pi G} \alpha \Omega^{-(d-4)} S_{a b} S_{c d} q^{a c} q^{b d}+O(\Omega) .
$$

It follows from eqs. (3) and (44) that the covector $l_{a}$ can be chosen in such a way that the following conditions are satisfied:

$$
\nabla_{a} l_{b}=O\left(\Omega^{\frac{d-4}{2}}\right), \quad l^{a} l_{a}=O\left(\Omega^{\frac{d-2}{2}}\right), \quad l_{a} n^{a}=1+O\left(\Omega^{\frac{d}{2}}\right)
$$

(for example, take $l_{a}=g_{a b} \bar{l}^{b}$, where $\bar{l}^{a}$ is a vector field on $\bar{M}$ such that $\bar{g}_{a b} \bar{l}^{a} \bar{l}^{b}=\bar{\nabla}_{a} \bar{l}^{b}=0$ and $\bar{l}^{a}(\mathrm{~d} \Omega)_{a}=1$ in a neighborhood of $\left.\partial \bar{M}\right)$. We assume from now on that $l_{a}$ has been chosen in this way.

¿From the defining relation for $S_{a b}$, together with eq. (62), we have

$$
\begin{aligned}
2 n^{a} \nabla_{[a} \nabla_{b]} l_{c} & =R_{a b c d} n^{a} l^{d} \\
& =C_{a b c d} n^{a} l^{d}+\frac{1}{2} S_{d b} n_{c} l^{d}-\frac{1}{2} S_{c b}-\frac{1}{2} l_{b} \nabla_{c} f+\frac{1}{2} g_{b c} l^{d} \nabla_{d} f .
\end{aligned}
$$

Contracting this equation with $S_{d e} q^{b d} q^{c e}$, and making use of the relations $S_{a b} n^{b}=-\nabla_{a} f$, $\nabla_{a} f=\kappa n_{a}, \kappa=O\left(\Omega^{(d-2) / 2}\right)$ and eq. (103) , gives

$$
\begin{aligned}
\Omega^{-(d-4)} S_{a b} S_{c d} q^{a c} q^{b d}= & -4 \Omega^{-(d-4)} n^{a}\left(\nabla_{[a} \nabla_{b]} l_{c}\right) S_{d e} q^{b d} q^{c e} \\
& +2 \Omega^{-(d-4)} C_{a b c f} n^{a} l^{f} S_{d e} q^{b d} q^{c e}+O(\Omega) .
\end{aligned}
$$

Thus, the task is to show that the right side of this equation can be written as a constant times the divergence of $P^{a}$, plus terms of order $\Omega$.

We now evaluate the right side of eq. (105) up to order $\Omega$, proceeding term by term and make heavy use of the drop-off conditions eq. (103) and eq. (69). For the second term in eq. (105) containing the Weyl tensor, we have ${ }^{25}$, using the symmetry of the Weyl tensor $C_{a b c d}=C_{c d a b}$,

$$
\begin{aligned}
2 \Omega^{-(d-4)} C_{a b c f} n^{a} l^{f} S_{d e} q^{b d} q^{c e}= & 2 \Omega^{-(d-4)+1}\left(\nabla_{[c} S_{f] b}\right) l^{f} S_{d e} q^{b d} q^{c e} \\
= & 2 \Omega \nabla_{[c}\left(\Omega^{-(d-4) / 2} S_{f] b}\right) l^{f} q^{c d} q^{b e}\left(\Omega^{-(d-4) / 2} S_{d e}\right) \\
& +(d-4) \Omega^{-(d-4)} n_{[c} S_{f] b} l^{f} q^{c d} q^{b e} S_{d e} \\
= & -\frac{(d-4)}{2} \Omega^{-(d-4)} S_{a b} S_{c d} q^{a c} q^{b d}+O(\Omega),
\end{aligned}
$$

\footnotetext{
${ }^{25}$ This term is of order $\Omega$ in 4 dimensions since $C_{a b c d}$ itself vanishes at $\mathscr{I}$ in 4 dimensions (see thm. 11 of [10]).
} 
where the identity [compare eq. (9) of [10]]

$$
\Omega \nabla_{[a} S_{b] c}+C_{a b c d} n^{d}=0
$$

has been used in the first line. We next turn to the first term on the right side of eq. (105). This can be written as

$$
\begin{aligned}
& -4 \Omega^{-(d-4)} n^{a}\left(\nabla_{[a} \nabla_{b]} l_{c}\right) S_{d e} q^{b d} q^{c e} \\
& =-4 \nabla_{a}\left(\Omega^{-(d-4)} S_{d e} q^{c e} q^{d[b} n^{a]} \nabla_{b} l_{c}\right)+4 \Omega^{-(d-4)}\left(\nabla_{a} S_{d e}\right) q^{c e} q^{d[b} n^{a]} \nabla_{b} l_{c}+O(\Omega) .(10
\end{aligned}
$$

In the second term on the right side, we may replace $\nabla_{a} S_{e d}$ by the expression $2 \nabla_{[a} S_{e] d}$, because

$$
\begin{aligned}
& \Omega^{-(d-4)}\left(\nabla_{e} S_{a d}\right) q^{c e} q^{d[b} n^{a]} \nabla_{b} l_{c} \\
& =\frac{1}{2} \Omega^{-(d-4)}\left[\left(\nabla_{e} \nabla_{d} f\right) q^{d b} q^{c e}-\left(\nabla_{e} S_{m}{ }^{m}\right) q^{c e} n^{b}-2 \nabla_{e}\left(l^{a} \nabla_{a} f\right) q^{c e} n^{b}\right] \nabla_{b} l_{c}+O(\Omega) \\
& =O(\Omega),
\end{aligned}
$$

where we have used that

$$
\nabla_{a} S_{m}^{m}=\nabla_{m} S_{a}^{m}
$$

by the Bianchi identities, that $S_{m}{ }^{m}=O\left(\Omega^{(d-2) / 2}\right)$ and that $\nabla_{a} f=\kappa n_{a}, \kappa=O\left(\Omega^{(d-2) / 2}\right)$. We can now apply the identity (107) to write the right side of eq. (108) as

$$
\begin{aligned}
= & -4 \nabla_{a}\left(\Omega^{-(d-4)} S_{d e} q^{c e} q^{d[b} n^{a]} \nabla_{b} l_{c}\right)-8 \Omega^{-(d-3)} C_{a e d f} q^{c e} q^{d[b} n^{a]} n^{f} \nabla_{b} l_{c}+O(\Omega) \\
= & -4 \nabla_{a}\left(\Omega^{-(d-4)} S_{d e} q^{c e} q^{d[b} n^{a]} \nabla_{b} l_{c}\right)-4 \Omega^{-(d-3)} C_{a e d f} n^{a} n^{f} \nabla^{d} l^{e} \\
& +4 \Omega^{-(d-3)} C_{a e d f} n^{a} n^{f} l^{e} n^{c} \nabla^{d} l_{c}+O(\Omega) \\
= & -4 \nabla_{a}\left(\Omega^{-(d-4)} S_{d e} q^{c e} q^{d[b} n^{a]} \nabla_{b} l_{c}\right)+4 \nabla^{d}\left(\Omega^{-(d-3)} C_{d f e a} n^{f} l^{e} n^{a}\right) \\
& +4 \Omega^{-(d-3)} C_{a e d f} n^{a} n^{f} l^{e} n^{c} \nabla^{d} l_{c}+4 \Omega^{-(d-3)} C_{a e d f} l^{e} n^{f} \nabla^{d} n^{a}+O(\Omega),
\end{aligned}
$$

where we have used the tracelessness and symmetries of the Weyl tensor and eqs. (103) and (69) in the second line, and where we have used [compare eq. (12) of [10]

$$
\Omega \nabla^{d} C_{a b c d}+C_{a b c d} n^{d}=0
$$

in the third line. Using Einstein's equation (53), the last term in the last line of eq. (111) is seen to be equal to $+2 \Omega^{-(d-4)} C_{a d e f} n^{a} l^{f} S^{d e}$, up to terms of order $\Omega$. Using eqs. (106) 
and eq. (107), this term can be further rewritten as

$$
\begin{aligned}
2 \Omega^{-(d-4)} C_{a d e f} n^{a} l^{f} S^{d e}= & 2 \Omega^{-(d-4)} C_{a d e f} n^{a} l^{f} S_{b c} q^{b d} q^{e c}+2 \Omega^{-(d-4)+1}\left(\nabla_{[e} S_{f] d}\right) l^{f} n^{e} l^{c} q^{b d} S_{b c} \\
& -2 \Omega^{-(d-4)+1}\left(\nabla_{[e} S_{f] d}\right) l^{f} l^{d} q^{e c} \nabla_{c} f \\
= & -\frac{(d-4)}{2} \Omega^{-(d-4)} S_{a b} S_{c d} q^{a c} q^{b d}+\Omega^{-(d-4)+1}\left(n^{e} \nabla_{e} S_{d f}\right) l^{f} l^{c} q^{b d} S_{b c} \\
& -\Omega^{-(d-4)+1}\left(\nabla_{f} S_{d e}\right) n^{e} l^{f} l^{c} q^{b d} S_{b c}+O(\Omega) \\
= & -\frac{(d-4)}{2} \Omega^{-(d-4)} S_{a b} S_{c d} q^{a c} q^{b d} \\
& +\Omega^{-(d-4)+1}\left(\nabla_{f} \nabla_{d} f+S_{d e} \nabla_{f} n^{e}\right) l^{f} l^{c} q^{b d} S_{b c}+O(\Omega) \\
= & -\frac{(d-4)}{2} \Omega^{-(d-4)} S_{a b} S_{c d} q^{a c} q^{b d}+O(\Omega) .
\end{aligned}
$$

The term $+4 \Omega^{-(d-3)} C_{a e d f} n^{a} n^{f} l^{e} n^{c} \nabla^{d} l_{c}$ on the right side of eq. (111) can be seen to be of order $\Omega$ by using the identities

$$
\begin{aligned}
n^{c} \nabla^{d} l_{c} & =\nabla^{d}\left(n^{c} l_{c}\right)-\left(\nabla^{d} n^{c}\right) l_{c}=O\left(\Omega^{\frac{d-2}{2}}\right) \\
C_{a e d f} n^{a} n^{f} & =-\frac{\Omega}{2}\left(n^{a} \nabla_{a} S_{e d}+\nabla_{e} \nabla_{d} f+\left(\nabla_{e} n^{a}\right) S_{a d}\right)=O\left(\Omega^{\frac{d-2}{2}}\right) .
\end{aligned}
$$

Substituting now eqs. (111) and (106) back into eq. (105), we obtain

$-(d-3) \Omega^{-(d-4)} S_{a b} S_{c d} q^{a c} q^{b d}=4 \nabla_{a}\left[\Omega^{-(d-4)}\left(S_{d e} q^{c e} q^{d[b} n^{a]} \nabla_{b} l_{c}-\Omega^{-1} C^{a b c d} n_{b} l_{c} n_{d}\right)\right]+O(\Omega)$,

from which eq. (102) follows immediately.

\section{B Conformal gauge choices}

In this appendix we review transformations related to the conformal completion of Minkowski spacetime, thereby eludicating our gauge choice (68) for the background geometry. Let us denote by $x^{\mu}$ the usual Cartesian coordinates of Minkowski spacetime $\left(\mathbb{R}^{d}, \tilde{\eta}_{a b}\right)$. Introducing the radial coordinate

$$
r=\sqrt{\sum_{\mu=1}^{d-1}\left(x^{\mu}\right)^{2}}
$$

and $t=x^{0}$, the Minkowski metric can be rewritten as

$$
\mathrm{d} \tilde{s}^{2}=-\mathrm{d} t^{2}+\mathrm{d} r^{2}+r^{2} \mathrm{~d} \sigma^{2}=\Omega^{-2}\left\{-\mathrm{d} T^{2}+\mathrm{d} \psi^{2}+\sin ^{2} \psi \mathrm{d} \sigma^{2}\right\},
$$

where $\mathrm{d} \sigma^{2}$ is the line element of the unit sphere $S^{d-2}$, and where

$$
\Omega=2 \cos \frac{T+\psi}{2} \cos \frac{T-\psi}{2} .
$$


The coordinates $T, \psi$ are defined by

$$
\frac{T+\psi}{2}=\tan ^{-1}(t+r), \quad \frac{T-\psi}{2}=\tan ^{-1}(t-r) .
$$

We view these relations as a map $\lambda$ from the portion $\bar{M}=\{-\pi<T \pm \psi<\pi, \psi \geq 0\}$ of the Einstein static universe $\mathbb{R} \times S^{d-1}$ to Minkowski spacetime $\mathbb{R}^{d}$. In other words,

$$
\bar{g}_{a b}=\Omega^{2} \lambda^{*} \tilde{\eta}_{a b}
$$

where $\bar{g}_{a b}$ is the metric of the Einstein static universe. The boundary of $\bar{M}$ corresponds to the conformal infinity of Minkowski spacetime. It is naturally divided into future/past timelike infinity, future/past null infinity $\mathscr{I}^{ \pm}$, and spatial infinity. The conformal factor $\Omega$ is well defined and smooth in a neighborhood of the null infinities $\mathscr{I}$, and vanishes there. The metric $\bar{g}_{a b}$ is conformally flat (implying $\bar{C}_{a b c d}=0$ ), but not flat, $\bar{S}_{a b} \neq 0$.

If we change

$$
\bar{g}_{a b} \rightarrow k^{2} \bar{g}_{a b}, \quad \Omega \rightarrow k \Omega,
$$

with $k$ a non-vanishing smooth scalar function defined in a neighborhood of $\bar{M}$ in the Einstein static universe which does not vanish at $\mathscr{I}$, then the physical metric remains unchanged, and the unphysical metric and the conformal factor remain smooth at $\mathscr{I}$. One may use this gauge freedom to make suitable "gauge choices" for the unphysical metric, and we will now discuss some of the choices that are being made in the main part of the paper. Let $B$ be a cross section of, say future, null infinity which does not intersect spatial infinity. Then it is possible to choose $\Omega$ so that $\bar{g}_{a b}$ is Minkowskian in an open neighborhood of $B$ not intersecting spatial infinity. This can be seen as follows:

Any neighborhood of the indicated form is contained in the causal future of some point in the interior of the spacetime, which of course, corresponds to the interior of a future directed lighcone $V^{+}$, whose apex we may assume to be at the origin,

$$
V^{+}=\left\{x^{\mu} \mid x^{\mu} x_{\mu}<0, x^{0}>0\right\} .
$$

A conformal factor, $\Omega$, defined on $V^{+}$such that $\bar{g}_{a b}$ is flat and Minkowskian and such that the gauge condition (68) is satisfied can be constructed as follows. Consider the map $\phi$ :

$$
\begin{aligned}
& \phi: x^{\mu} \rightarrow x^{\prime \mu}=\frac{a^{\mu}+b^{\mu} x^{\lambda} x_{\lambda}+2 q^{\mu \nu} x_{\nu}}{2 b^{\nu} x_{\nu}} \\
& a^{\mu}=(1,-1,0, \ldots, 0), \quad b^{\mu}=(1,1,0, \ldots, 0), \quad q^{\mu \nu}=\eta^{\mu \nu}+a^{(\mu} b^{\nu)},
\end{aligned}
$$

which maps points of the interior of $V^{+}$bijectively into points in the "right wedge" $W$ of Minkowski spacetime

$$
W=\left\{x^{\mu}\left|x^{1} \geq\right| x^{0} \mid\right\} .
$$

The portion of $\mathscr{I}^{+}$lying in the causal futuer of $V^{+}$corresponds, under the map $\phi$, to the "upper horizon" of $W$, defined by $\partial W^{+}=\left\{x^{\mu} \mid b^{\mu} x_{\mu}=0, x^{0}>0\right\}$. The cross section 
of $\mathscr{I}^{+}$corresponding to the lightrays outgoing from the apex of $V_{+}$corresponds to the "edge" $\left(x^{0}=0=x^{1}\right)$ of the wedge, whereas the lighrays themselves are represented by the null curves generated by $a^{\mu}\left(\partial / \partial x^{\mu}\right)^{a}$ on the "lower horizon" of $W$, given by $\partial W^{-}=$ $\left\{x^{\mu} \mid a^{\mu} x_{\mu}=0, x^{0}<0\right\}$. We find that this map $\phi$ is a conformal isometry of Minkowski spacetime with conformal factor

$$
\Omega=b_{\mu} x^{\mu}
$$

i.e., the background metric $\bar{g}_{a b}=\Omega^{2} \phi^{*} \tilde{\eta}_{a b}$ is Minkowskian. The quantities $\bar{f}, \bar{n}^{a}$ associated with this choice of conformal factor are

$$
\bar{f}=0, \quad \bar{n}^{a}=b^{\mu}\left(\frac{\partial}{\partial x^{\mu}}\right)^{a}, \quad \bar{\nabla}_{a} \bar{n}^{b}=0 .
$$

Thus, the conformal transformation (124) with conformal factor (126) satisifies our gauge condition (68).

An awkward feature of the map $\phi$ is that it is not globally defined on the boundary of $V^{+}$, for the single null generator corresponding to $x^{\prime \mu}=\lambda b^{\mu}, \lambda>0$ of the boundary of $\mathrm{V}^{+}$is mapped to infinity. Consequently, there is also a single corrsponding generator of $\mathscr{I}^{+}$which is not represented as a corresponding generator of $\partial W^{+}$, or, stated differently, is mapped to the null generator at 'infinity' of the upper horizon $\partial W^{+}$(corresponding to $b_{\mu} x^{\mu}=0, x^{0}>0$ but $\left.x^{\mu=2, \cdots, d-1} \rightarrow \pm \infty\right)$. Consequently, the cross sections of $\mathscr{I}^{+}$within the causal future of $V^{+}$now corrspond to non-compact cross sections of the upper horizon of $W$ (of topolgy $\mathbb{R}^{d-2}$ ). This feature of the conformal embedding $\phi$ has the undesirable consequence that the integrals in section 5 over cross sections of $\mathscr{I}^{+}$inside $V^{+}$are now integrals over a noncompact set and therefore the convergence issue must be addressed. An example of such an integral is $\int_{\mathscr{S}} \Theta$, where $\Theta$ is the symplectic potential $(d-1)$-form introduced above in eq. (63), and where $\mathscr{S}$ is a segment of scri. We will now explain how the convergence issue is dealt with in this example.

For this purpose, it is useful to introduce another conformal transformation:

$$
\psi: x^{\mu} \rightarrow x^{\prime \mu}=\frac{(x+t)^{\nu}(x+t)_{\nu} t^{\mu}+2(x+t)^{\mu}}{(x-t)^{\lambda}(x-t)_{\lambda}}, \quad t^{\mu}=(1,0,0, \ldots, 0),
$$

which maps points in $V^{+}$into points of the interior of a double cone $K$ of Minkowski spacetime,

$$
K=\left\{x^{\mu}|| x^{0} \mid+r \leq 1\right\} .
$$

This map also provides a conformal isometry of Minkowski spacetime with conformal factor

$$
\Omega^{\prime}=-(x-t)^{\mu}(x-t)_{\mu},
$$

rendering the metric $\bar{g}^{\prime}{ }_{a b}=\Omega^{\prime 2} \psi^{*} \tilde{\eta}_{a b}$ Minkowskian in the portion of spacetime corresponding to the interior of the future lightcone $V^{+}$. The derivative operator $\bar{\nabla}_{a}^{\prime}$ compatible 
with $\bar{g}_{a b}^{\prime}=\Omega^{\prime 2} \psi^{*} \tilde{\eta}_{a b}$ is simply equal to the coordinate derivative operator $\partial / \partial x^{\mu}$ associated with Cartesian coordinates. The portion of $\mathscr{I}^{+}$that can be reached from within $V^{+}$ corresponds precisely to the points $x^{\mu}$ in $K$ such that $\Omega^{\prime}=0$, i.e., the "upper cap" of the double cone $K$, defined by $\partial K^{+}=\left\{x^{\mu} \mid x^{0}+r=1, x^{0}>0\right\}$. Future timelike infinity corresponds to the apex $x^{\mu}=t^{\mu}$ of the upper cap. The apex of $V^{+}$corresponds to the apex of the lower cap of $K$ given by $x^{\mu}=-t^{\mu}$, and the lightrays going out from the apex of $V_{+}$correspond to the null generators of the lower cap, $\partial K^{-}$, of $K$. We should note that this choice of conformal factor $\Omega^{\prime}$ does not satisfy our gauge condition (68), as the quantities $\bar{n}^{\prime a}, \bar{f}^{\prime}$ associated with $\Omega^{\prime}$ satisfy

$$
\bar{f}^{\prime}=4, \quad \bar{n}^{\prime a}=-2(x-t)^{\mu}\left(\frac{\partial}{\partial x^{\mu}}\right)^{a}, \quad \bar{\nabla}_{a}^{\prime} \bar{n}^{\prime b}=-2 \delta_{a}^{b} .
$$

The advantage of the conformal transformation (128) with conformal factor $\Omega^{\prime}$ is however that it preserves the compactness of cross sections of $\mathscr{I}^{+}$. In fact, the cross section of $\mathscr{I}^{+}$corresponding to the outgoing lightrays from the apex of $V^{+}$is represented by the "belt" of $K,\left(x^{0}=0, r=1\right)$, and all other cross sections to the future of this particular one are given by cross sections of the upper cap, and are therefore topological spheres.

We compose the two maps $\phi$ and $\psi$ to the map $\sigma=\phi \circ \psi^{-1}: K \rightarrow W$. Under the map $\sigma$ the set $\partial W^{+}$is identified with the upper cap $\partial K^{+}$of the double cone $K$. If we denote $\bar{g}_{a b}$ the Minkowskian metric of $W$, then under this map, $\bar{g}_{a b}$ gets mapped to the metric $k^{-2} \bar{g}_{a b}^{\prime}$, where $\bar{g}_{a b}^{\prime}$ is the Minkowskian metric on $K$, and where $k$ is calculated to be

$$
k=(x-t+b)^{\mu}(x-t+b)_{\mu} .
$$

The conformal factor $k$ vanishes on the single null generator emanating from future timelike infinity (represented by the point $x^{\mu}=t^{\mu}$ of $\partial K^{+}$) parallel to $b^{\mu}$. This generator corrsponds to the generator at infinity in $\partial W^{+}$under the map $\sigma$, and the vanishing of $k$ on this generator is a reflection of this fact ${ }^{26}$.

Returning to the example integral, let $\mathscr{S}$ be a segment of $\mathscr{I}^{+}$, viewed as a subset of the upper horizon $\partial W^{+}$of $W$, with non-compact cross-sections, i.e., $\mathscr{S}$ has topology $\mathbb{R}^{d-2} \times I$, where $I$ is a compact interval. Let $\mathscr{S}^{\prime}$ be the segment of $\partial K^{+}$corresponding to $\mathscr{S}$ under $\sigma$, i.e., $\sigma\left(\mathscr{S}^{\prime}\right)=\mathscr{S}$. Then $\mathscr{S}^{\prime}$ has compact cross sections homeomorphic to $S^{d-2}$ as $\partial K^{+}$does. By eq. (66) the symplectic form satisfies

$$
\int_{\mathscr{S}} \Theta=\int_{\mathscr{S}^{\prime}} \Theta^{\prime}+\delta \int_{\mathscr{S}^{\prime}} \Pi^{\prime}
$$

where $\Theta^{\prime}$ is given in terms of $\Omega^{\prime}=k^{-1} \sigma^{*} \Omega, g^{\prime}{ }_{a b}=k^{2} \sigma^{*} g_{a b}$ and $\tau_{a b}^{\prime}=k^{\frac{d-2}{2}} \sigma^{*} \tau_{a b}$ by a formula analogous to (63), and where $\Pi^{\prime}$ is given by eq. (67). Using that $n^{\prime a}=-2\left(x^{\mu}-\right.$

\footnotetext{
${ }^{26} \mathrm{In}$ other words, $\partial K^{+}$is the comactification of $\partial W^{+}$.
} 
$\left.t^{\mu}\right)\left(\partial / \partial x^{\mu}\right)^{a}$ by eq. (131), as well as the above expression (132) for the conformal factor $k$, one immediately finds that

$$
k^{-1} n^{\prime a} \nabla_{a}^{\prime} k=-2
$$

at points of $\partial K^{+}$. Inserting this into the definition of $\Pi^{\prime}$, one gets

$$
\Pi^{\prime}=\frac{(d-2)}{2^{5} \pi G} \Omega^{\prime-(d-2)}\left(g^{\prime}-\bar{g}^{\prime}\right)_{c d} q^{\prime c e} q^{\prime d f}\left(g^{\prime}-\bar{g}^{\prime}\right)_{e f}{ }^{(d-1)} \epsilon^{\prime} .
$$

Now $\Pi^{\prime}$ and $\Theta^{\prime}$ are finite at $\mathscr{I}$ as a result of our asymptotic flatness conditions, and the integrals on the right side of eq. (133) are over a compact set, $\mathscr{S}^{\prime}$ of $\mathscr{I}$, (viewed as a subset of the upper cap $\partial K^{+}$via the map $\sigma$ ). This shows that the integral of the symplectic potential over $\mathscr{S}$ appearing on the left side of eq. (133) is convergent. The same kind of argument can be made for other integrals appearing above in section 5 .

\section{Asymptotic translations}

We finally discuss the space of translational asymptotic symmetries $\xi^{a}$ of the form $\xi^{a}=$ $\alpha n^{a}-\Omega \nabla^{a} \alpha$ in $d>4$ dimensions. As discussed in section 5, in order for such a vector field to be an asymptotic symmetry, we must satisfy eqs. (78), (79) and (801), which we here repeat for convenience:

$$
\begin{aligned}
\nabla_{a} \nabla_{b} \alpha-\Omega^{-1} g_{a b} n^{c} \nabla_{c} \alpha & =O\left(\Omega^{\frac{d-4}{2}}\right), \\
\nabla^{b}\left(\Omega^{-1} n^{a} \nabla_{a} \alpha\right) & =O\left(\Omega^{\frac{d-4}{2}}\right), \\
\Omega^{-1} n^{b} \nabla_{b}\left(\Omega^{-1} n^{a} \nabla_{a} \alpha\right) & =O\left(\Omega^{\frac{d-4}{2}}\right) .
\end{aligned}
$$

These equations hold in any conformal gauge choice satisfying eq. (68). Actually, by our asymptotic flatness conditions, if these conditions are satisfied for one given asymptotically flat metric, they are satsified for any asymptotically flat metric (satisfying our gauge choice (68)). In order to analyze these equations, we may therefore choose $g_{a b}$ to be equal to our Minkowskian background metric $\bar{g}_{a b}=\Omega^{2} \phi^{*} \tilde{\eta}_{a b}$, where $\phi$ is the conformal map $V_{+} \rightarrow W$ defined above in eq. (124), and where the conformal factor is defined in eq. (126). The derivative operator $\bar{\nabla}_{a}$ is then given by the coordinate derivative operator $\partial / \partial x^{\mu}$, and the associated quantity $\bar{n}^{a}$ is given by $b^{\mu}$ in Cartesian coordinates. Inserting these expressions and going to Cartesian coordinates, eqs. (136), (137) and (138) become, respectively

$$
\begin{aligned}
\partial_{\mu} \partial_{\nu} \alpha+2 \eta_{\mu \nu} w^{-1} \partial_{v} \alpha & =O\left(w^{\frac{d-4}{2}}\right) \\
\partial_{\mu}\left(w^{-1} \partial_{v} \alpha\right) & =O\left(w^{\frac{d-4}{2}}\right) \\
w^{-1} \partial_{v}\left(w^{-1} \partial_{v} \alpha\right) & =O\left(w^{\frac{d-4}{2}}\right),
\end{aligned}
$$


where we have now set $w=x^{0}-x^{1}$ and $v=x^{0}+x^{1}$, and where we remember that the location of null infinity corresponds to $\Omega=-w=0$ in our gauge.

Let us first check that the timelike translational Killing vector fields

$$
\tau^{a}=\tau^{\mu}\left(\frac{\partial}{\partial x^{\mu}}\right)^{a}, \quad \tau^{\mu}=\left(\tau^{0}, \tau^{1}, \ldots, \tau^{d-1}\right)=\text { const }
$$

in Minkowski spacetime give rise to solutions of eqs. (139). Under the identification provided by the map $\phi$, these vector fields correspond to

$$
\xi^{a}=\phi^{*} \tau^{a}=\left(\alpha b^{\mu}-\Omega \partial^{\mu} \alpha\right)\left(\frac{\partial}{\partial x^{\mu}}\right)^{a}=\alpha \bar{n}^{a}-\Omega \bar{\nabla}^{a} \alpha
$$

where

$$
\alpha=\frac{1}{2}\left(b^{\lambda} \tau_{\lambda} x^{\mu} x_{\mu}+2 q_{\mu \nu} \tau^{\mu} x^{\nu}+a^{\mu} \tau_{\mu}\right) .
$$

By construction, since $\phi$ is a conformal isometry of Minkowski spacetime, we must have

$$
\partial_{\mu} \partial_{\nu} \alpha+2 \eta_{\mu \nu} w^{-1} \partial_{v} \alpha=0
$$

which can also be verified explicitly. This shows eq. (139), and the other equations (140) and (141) follow by dotting $b^{\mu}$ into this equation. It is not difficult to see that (in $d>4$ ), any other solution $\alpha$ to eqs. (139), (140) and (141) is given by

$$
\alpha=\alpha_{0}+O\left(\Omega^{\frac{d}{2}}\right)
$$

where $\alpha_{0}$ is given by eq. (144). Inserting this into the definition of $\chi_{a b}$, eq. (77), we see that

$$
\chi_{a b}=\chi_{0 a b}+O(1) n_{a} n_{b}+O(\Omega),
$$

where $\chi_{0 a b}$ is defined by eq. (77), with $\alpha$ replaced by $\alpha_{0}$. Now the integrand of the flux associated with an asymptotic symmetry is given by (see eq. (71) ) $N_{a b} \chi^{a b}$ up to numerical factors, and $N_{a b} n^{b}=0$. Thus, the second term in eq. (147) does not contribute to the flux. This shows that the $\alpha_{0}$ given by eq. (144) are essentially the only solutions to eqs. (139), in the sense that any other solution will give rise to the same flux. Hence, the vector space of infinitesimal asymptotic translations is $d$-dimensional in $d>4$, consists of the vector fields $\xi^{a}=\alpha n^{a}-\Omega \nabla^{a} \alpha$, with $\alpha$ given by eq. (144) in the gauge that we are working in.

Let us finally characterize the $\alpha$ corresponding to a future directed timelike translational Killing fields $\tau^{\mu}$ of Minkowski spacetime. A point $x^{\mu}$ of $\mathscr{I}^{+}$corresponds to a point on $\partial W^{+}$under the map $\phi$, so we have $b^{\mu} x_{\mu}=0$ and $x^{\lambda} x_{\lambda}=q_{\mu \nu} x^{\mu} x^{\nu}$ for such points. Also, since $\tau^{\mu}$ is future pointing timelike, we have $a^{\mu} \tau_{\mu} b^{\nu} \tau_{\nu}>q_{\mu \nu} \tau^{\mu} \tau^{\nu}$. Using this, and the inequality obtained by expanding out the relation

$$
0 \leq q_{\mu \nu}\left[\left(b^{\lambda} \tau_{\lambda}\right)^{-1 / 2} \tau^{\mu}+\left(b^{\lambda} \tau_{\lambda}\right)^{1 / 2} x^{\mu}\right]\left[\left(b^{\sigma} \tau_{\sigma}\right)^{-1 / 2} \tau^{\nu}+\left(b^{\sigma} \tau_{\sigma}\right)^{1 / 2} x^{\nu}\right]
$$


one easily finds that $\alpha>0$ on $\partial W^{+}$. Conversely, if $\tau^{\mu}$ is such that $\alpha>0$ on $\partial W^{+}$, then one sees by the same argument that it must be future directed timelike. Thus, future directed timelike translational Killing fields correspond to asymptotic symmetries $\xi^{a}$ with $\alpha>0$ on $\mathscr{I}^{+}$.

\section{References}

[1] R. Arnowitt, S. Deser and C. W. Misner, in Gravitation: An introduction to current research, ed. L. Witten, Wiley, New York (1962)

[2] H. Bondi, M. G. J. van der Burg and A. W. K. Metzner, Proc. R. Soc. London A269, $21-52(1962)$

[3] A. Trautman, Bull. Acad. Pol. Sci., Ser. Sci., Math., Astron., Phys. 6, 407 (1958)

[4] R. Sachs, Proc. R. Soc. London A270, 103 (1962)

[5] M. A. Awada, G. W. Gibbons, and W. T. Shaw, Ann. Phys. 171 52, (1986)

[6] T. Dray and M. Streubel, Class. Quantum. Grav. 1, 15 (1984)

[7] R. Penrose, Proc. R. Soc. London A284, 159 (1965)

[8] L. Tamburino and J. Winicour, Phys. Rev. 150, 1039 (1966)

[9] R. Geroch, J. Math. Phys. 13, 956 (1972)

[10] R. Geroch, in Asymptotic structure of spacetime, ed. F. Esposito and L. Witten, Plenum Press, New York (1977)

[11] A. Ashtekar and M. Streubel, Proc. R. Soc. London A376, 585 (1981)

[12] R. Geroch and B. C. Xanthopoulos, J. Math. Phys. 19, 714 (1978)

[13] T. Regge and C. Teitelboim, Ann. Phys. 88, 286 (1974)

[14] R. M. Wald and A. Zoupas, Phys. Rev. D61, 084027 (2000)

[15] A. Ashtekar, L. Bombelli and O. Reula, in: Mechanics, Analysis, and Geometry: 200 Year after Lagrange, ed. M. Francaviglia, Vol. 376, Elsevir, Amsterdam (1991)

[16] R. M. Wald, General Relativity, University of Chicago Press, Chicago (1984)

[17] S. W. Hawking and G. F. R. Ellis, The large scale structure of space-time (Cambridge University Press, Cambridge, 1973) 
[18] L. Tamburino and J. Winicour, Phys. Rev. 150, 1039 (1966)

[19] S. Hollands and A. Ishibashi, work in progress.

[20] R. C. Myers and M. J. Perry, Annals Phys. 172, 304 (1986) 